\title{
Integrated analysis of co-expression, conserved genes and gene families reveal core regulatory network of heat stress response in Cleistogenes songorica, a xerophyte perennial desert plant
}

\author{
Qi Yan, Xifang Zong, Fan Wu, Jie Li, Tiantian Ma, Yufeng Zhao, Qian Ma, Penglei Wang, Yanrong Wang and \\ Jiyu Zhang ${ }^{*}$ (D)
}

\begin{abstract}
Background: As global warming continues, heat stress (HS) is becoming an increasingly significant factor limiting plant growth and reproduction, especially for cool-season grass species. The objective of this study was to determine the transcriptional regulatory network of Cleistogenes songorica under HS via transcriptome profiling, identify of gene families and comparative analysis across major Poaceae species.

Results: Physiological analysis revealed significantly decreased leaf relative water content (RWC) but increased proline (Pro) content in C. songorica under $24 \mathrm{~h}$ of HS. Transcriptome profiling indicated that 16,028 and 14,645 genes were differentially expressed in the shoots and roots of $C$. songorica under HS, respectively. Two subgenomes of $C$. songorica provide equal contribution under HS on the basis of the distribution and expression of differentially expressed genes (DEGs). Furthermore, 216 DEGs were identified as key evolutionarily conserved genes involved in the response to HS in C. songorica via comparative analysis with genes of four Poaceae species; these genes were involved in the 'response to heat' and 'heat acclimation'. Notably, most of the conserved DEGs belonged to the heat-shock protein (HSP) superfamily. Similar results were also obtained from co-expression analysis. Interestingly, hub-genes of co-expression analysis were found to overlap with conserved genes, especially heat-shock protein (HSP). In C. songorica, 84 HSP and 32 heat-shock transcription factor (HSF) genes were identified in the allotetraploid C. songorica genome, and might have undergone purifying selection during evolutionary history based on syntenic and phylogenetic analysis. By analysing the expression patterns of the CSHSPs and CSHSFs, we found that the transcript abundance of $72.7 \%$ of the CSHSP genes and of $62.5 \%$ of the CSHSF genes changed under heat stress in both the shoots and roots. Finally, a core regulatory network of HS was constructed on the basis of the CSHSP, CSHSF and other responsive genes in C. songorica. (Continued on next page)
\end{abstract}

\footnotetext{
* Correspondence: zhangjy@|zu.edu.cn

State Key Laboratory of Grassland Agro-ecosystems, Key Laboratory of Grassland Livestock Industry Innovation, Engineering Research Center of Grassland Industry, Ministry of Education, College of Pastoral Agriculture Science and Technology, Lanzhou University, Lanzhou 730020, People's Republic of China
}

(c) The Author(s). 2020 Open Access This article is licensed under a Creative Commons Attribution 4.0 International License, which permits use, sharing, adaptation, distribution and reproduction in any medium or format, as long as you give appropriate credit to the original author(s) and the source, provide a link to the Creative Commons licence, and indicate if changes were made. The images or other third party material in this article are included in the article's Creative Commons. licence, unless indicated otherwise in a credit line to the material. If material is not included in the article's Creative Commons licence and your intended use is not permitted by statutory regulation or exceeds the permitted use, you will need to obtain permission directly from the copyright holder. To view a copy of this licence, visit http://creativecommons.org/licenses/by/4.0/ The Creative Commons Public Domain Dedication waiver (http://creativecommons.org/publicdomain/zero/1.0/) applies to the data made available in this article, unless otherwise stated in a credit line to the data. 
(Continued from previous page)

Conclusions: Regulatory network and key genes were comprehensively analysed and identified in C. songorica under HS. This study improves our knowledge of thermotolerance mechanisms in native grasses, and also provides candidate genes for potential applications in the genetic improvement of grasses.

Keywords: Heat stress, Cleistogenes songorica, RNA sequencing, Conserved heat-responsive genes, Co-expression, Regulatory network, HSP, HSF

\section{Background}

It has been widely confirmed that heat stress (HS) is one of the major limiting factors for plant growth and productivity worldwide [1]. Global temperature is likely to rise by $3-6^{\circ} \mathrm{C}$ in the twenty-first century because of global warming, and the duration, intensity and frequency of high-temperature periods will increase during the vegetative stages and reproductive periods of plants $[2$, 3]. Therefore, it is important to determine the genomic, transcriptomic and physiological mechanisms of heat tolerance in plants, which are of great importance to accelerate crops and forages breeding aiming at thermotolerance improvement. Cleistogenes songorica, a C4 plant, is a desert grass that distributed widely in the wild lands in the northwest part of China [4]. C. songorica can grow in semi-arid, arid and desert regions where the annual rainfall ranges from 100 to $150 \mathrm{~mm}$. It is known to have novel drought stress adaptation strategies and contains rich pools of stress tolerance genes based on transcriptome profiling and identification of transcription factor and protein family [5-9]. Increasing amounts of evidence suggested that stress tolerance genes of native grass could facilitate tolerance improvement of plant for drought, heat, salt stress. For example, overexpression of the heat-shock transcription factors 1 (HSFs) of resurrection plant Boea hygrometrica leads to increased thermotolerance and retarded growth in transgenic Arabidopsis and tobacco [10]. Co-overexpression of $Z x N H X$ and $Z x V P 1-1$ genes from Zygophyllum xanthoxylum enhances enhanced salinity and drought tolerance in Lotus corniculatus [11]. In previous study, we also confirmed that overexpression of functional genes form $C$. songorica also improved tolerance to drought and salt in both alfalfa and Arabidopsis, such as CSLEA and CsALDH [68]. However, the transcriptional regulatory network and potential candidate genes involved in HS response of native plant is not well documented, especially those of grass species.

Some studies have suggested that plants have developed complex regulatory networks at the biochemical, physiological and transcriptional levels to cope with HS; (1) The first response of plants to HS involves an increase in membrane fluidity of the plasmalemma. (2) This leads to the production of secondary messengers and chemical signals such as calcium in the cytoplasm.
Mitogen-activated protein kinases (MAPKs) and calcium-dependent protein kinases (CDPKs) are in turn immediately regulated by the $\mathrm{Ca}^{2+}$ influx. (3) To maintain the water balance and osmotic adjustment in the cell, these signalling components are further transmitted to the nucleus, (4) resulting in the induction of antioxidants, electron transport and solutes that function osmotically in the cytoplasm, such as ascorbic acid, proline (Pro) and glutathione [12].

Along with the rapid development of sequencing technology, transcriptomic analysis is an extremely powerful tool for investigating plant molecular responses to abiotic stress, the knowledge of which could be further used to improve plant tolerance through molecular engineering. It has been widely used to identify heat-responsive genes in model plants, moss species, crop species and turfgrass species, such as Arabidopsis [13], rice [14], pepper (Capsicum annuum L.) [15], Physcomitrella patens [16], and Agrostis scabra [17]. Many HS-responsive genes have been identified in transcriptional regulatory network of model plant, including heat shock proteins (HSPs) and HSFs $[18,19]$. HSPs play a key role in signal transduction and gene regulation under HS and can also interact with other stress response mechanisms [20]. HSPs protein could be divided into different groups on the basis of their molecular weight: for example, there are small HSP (sHSP), HSP70, HSP90 and HSP100 groups [21]. HSPs have been identified in plant species such as Arabidopsis, rice, Brachypodium distachyon and Zea mays, and the functions of these HSPs have been determined [21-23]. For instance, overexpression of OsHSP26 can improve the tolerance of HS by protecting the photosystems [24]. Furthermore, HSPs can regulate the expression of $H S F \mathrm{~s}$, which largely participate in gene regulation under HS. HSF transcription factor family can be divided into $\mathrm{A}, \mathrm{B}$ and $\mathrm{C}$ groups and play important roles in signalling pathways and transcriptional regulation [25]. For example, as compared with wild-type plants, transgenic AtHsfA1 plants are significantly more tolerant to heat and drought stress [26]. HSP70/HSP9O decreases the inhibition of HSFA1s under HS, resulting in the activation or reduction in gene expression by downstream transcription factors (TFs). Similarly, mutant Arabidopsis HSFA1 plants are sensitive to HS [27]. Dehydration-responsive element binding protein $2 A$ 
(DREB2A), a direct target gene of HSFA1, is a key gene involved in post-translational regulation under $\mathrm{HS}$. $D R E B 2 A$ can positively regulate $H S F A 3$ gene expression by interacting with other genes, such as the subunits of nuclear factor $Y$ A2 (NF-YA2), NF-YB3 and DNA polymerase II subunit B3-1 (DPB3-1) [28, 29]. HSFA3 can activate the expression of downstream genes, including those encoding chaperones and enzymes. Overexpression or mutation of HSFA3 in A. thaliana was shown to increase or decrease the tolerance of transgenic plants, respectively $[29,30]$. In addition, HSFA2 and HSFA 7 act as target genes of HSFA1, which involves two adjustment processes: HSFA2 and HSFA7 can be directly positively regulated by $H S F A 1$, and $H S F A 1$ negatively regulates $H S F A 2$ and $H S F A 7$ by inducing the expression of $H S F B$. Strikingly, $H S F B$ not only is controlled by HSFA 1 but also inhibits the activity of HSFA1 via a negative feedback loop [18].

In the present study, we performed a genome-wide transcriptomic analysis of $C$. songorica, comparative analysis across major Poaceae species, co-expression network, and identification of gene families under HS. We aimed to address the following main questions: (i) How many/which genes exhibit significant changes in expression in C. songorica under stress? (ii) What key Gene Ontology (GO) terms and Kyoto Encyclopedia of Genes and Genomes (KEGG) pathways are enriched? (iii) How many/which genes play key roles and are conserved or co-expression under HS in the Poaceae? Our study provided new insights into the regulatory mechanism of $C$. songorica under HS and identified candidate genes associated with HS, these genes may be used for molecular breeding new varieties with thermotolerance.

\section{Results}

\section{Phenotype and physiology responses of $C$. songorica} under HS

To study the physiological changes caused by HS, nineweek-old seedlings of $C$. songorica were placed in three growth chamber with a $40{ }^{\circ} \mathrm{C}$ temperature during the day and night for $0 \mathrm{~h}, 6 \mathrm{~h}, 12 \mathrm{~h}, 24 \mathrm{~h}, 36 \mathrm{~h}, 48 \mathrm{~h}$ or $72 \mathrm{~h}$. Under high-temperature exposure, the relative water content (RWC) gradually decreased and significantly differed $(p<0.05)$ at $24 \mathrm{~h}$ and a few leaves began to curl in C. songorica (Additional file 1: Fig. S1a-b). Remarkably, compared with that at $0 \mathrm{~h}$, the RWC after $48 \mathrm{~h}$ of treatment decreased by $36.4 \%$. Under high temperature treatment, leaf temperature was stably increased with decreasing water content, whereas soil temperature increased more rapidly in C. songorica (Additional file 1: Fig. S1h-i). Leaf and soil temperature increased by 1.35 and 1.15 -fold at $6 \mathrm{~h}$ treatment, respectively. Additionally, the Pro and malondialdehyde (MDA) contents gradually increased under HS and markedly increased at $6 \mathrm{~h}$ and
$48 \mathrm{~h}$, respectively, in $C$. songorica seedlings; compared with those at $0 \mathrm{~h}$, the Pro and MDA contents increased by 14.9 - and 1.46-fold, respectively (Additional file 1: Fig. S1c-d). Moreover, we also analysed the changes in photosynthesis traits under HS. The results indicated that injury to the photosystems occurred very quickly under HS. The Fv/Fm, rETRmax and quantum yield of photosystem II (PSII (Y(II)) significantly decreased $(p<0.05)$ to $55.96,64.25$ and $60.07 \%$ of their respective values at $0 \mathrm{~h}$, respectively, after $6 \mathrm{~h}$ of treatment (Additional file 1: Fig. S1e-g).

\section{Identification of DEGs in the subgenome of $C$. songorica}

To reveal the changes in transcript levels in the roots and shoots of $C$. songorica under HS, samples collected at $0 \mathrm{~h}$ and $24 \mathrm{~h}$ were subjected to genome-wide RNA sequencing (RNA-seq) analysis. A total of $657,826,480$ clean reads from 12 samples (two treatments, two tissues, three biological replicates) were mapped to the $C$. songorica genome after filtering and quality control were performed. The Q30 contents and mapping percentages were mostly greater than 91 and $80 \%$, respectively (Additional file 2: Table S1). To validate the RNA-seq data, nine genes were chosen for qRT-PCR, including HSFA1-2, HSFA3-1, HSFA3-2, HSFA9-1, HSFA9-2, HSP90-6, HSP70-7, JUB1 and NF-YB3-2. The results indicated that the expression profiles of these gene were coincident with gene expression of RNA-seq (Fig. 9b). In total, 22,362 genes were significantly differentially expressed under HS (log2(fold change) $\geq 1$; FDR $\leq 0.01$ ) in the roots and shoots. Among these genes, the number of DEGs in the shoots was greater than that in the roots $(16,028$ and 14,645 DEGs, respectively; Fig. 1a; Additional file 3: Table S2). Strikingly, the numbers of upregulated and downregulated DEGs were significantly different. For example, there were 6711 upregulated DEGs and 9317 downregulated DEGs in the C. songorica shoots. A similar trend was also found in the roots. By comparing the 8311 DEGs common to the different organs, we observed a further general conservation of expression, with the expression of 7825 of the genes being regulated in the same way in the shoots and roots and that of 486 genes displaying opposite regulatory patterns (Fig. 1b). A total of 4737 downregulated and 3088 upregulated DEGs were found both in shoots and roots. As shown in Fig. 1c, the gene expression pattern was obviously changed under HS and DEGs could be grouped into four expression clusters; cluster I and cluster IV consisted of DEGs whose expressions were upregulated and downregulated both in the shoots and roots, respectively (Fig. 1c).

C. songorica is an allotetraploid plant, so we also analysed the distribution of DEGs in the two subgenomes of C. songorica. The results indicated that the DEGs were 


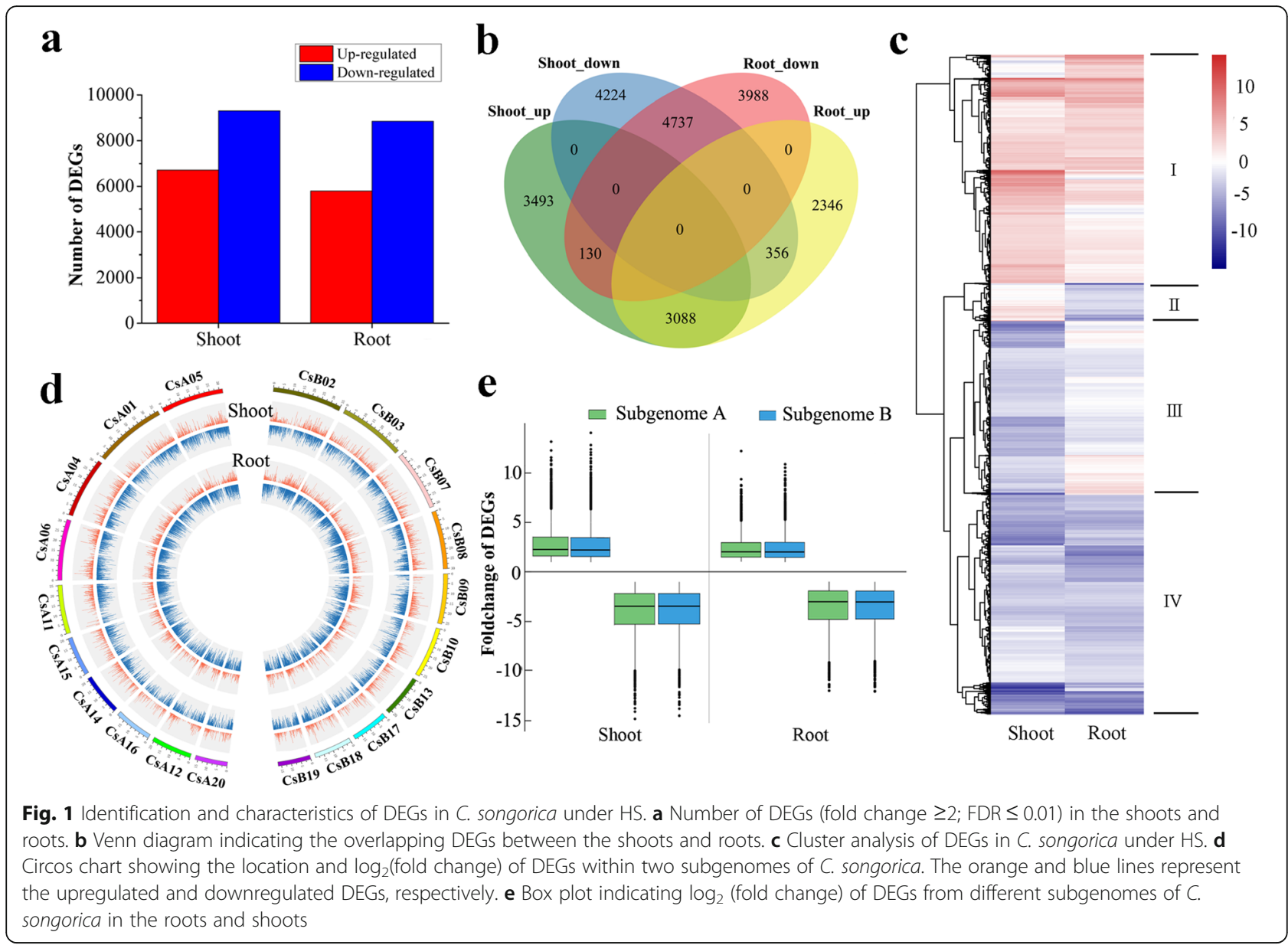

uniformly distributed in the two subgenomes of C. songorica. A detailed assessment of the numbers of DEGs between the subgenome A and subgenome B conditions for each organ confirmed this observation; in the shoots, there were 2716 DEGs whose expression was upregulated (4255 downregulated DEGs) and 2874 DEGs whose expression was upregulated (4298 downregulated DEGs) in subgenome A and subgenome B, respectively (Fig. 1d; Additional file 3: Table S2). Similar trends were identified in the roots of $C$. songorica. We also analysed the expression levels of the DEGs on the basis of fold changes and found that the expression of the DEGs was similar between subgenome $\mathrm{A}$ and subgenome $\mathrm{B}$ of $C$. songorica (Fig. 1e).

\section{GO enrichment analysis of DEGs}

To identify the major functions of the heat-responsive genes in C. songorica, we performed a GO enrichment analysis of the DEGs in the shoots and roots of $C$. songorica. On the basis of the GO enrichment results, we found that the majority of DEGs were involved in stressrelated categories, including 'response to stimulus', 'nucleic acid binding transcription factor activity', 'organelle', and 'developmental process'. Notably, the biological process category revealed differences in the number of associated DEGs between the two organs, with greater expression in the shoots than in the roots (Additional file 1: Fig. S2). To identify the functional enrichment categories of the DEGs further, we used a $p$ value $<0.05$ to filter the significantly enriched $\mathrm{GO}$ categories. We also compared the numbers of GO categories between the different organs and the direction of regulation. Four comparisons were performed: shoot_up (upregulated genes in the shoots), shoot_down (downregulated genes in the shoots), root_up (upregulated genes in the roots) and root_down (downregulated genes in the roots) (Fig. 2).

One biological process GO term was found in the shoot and root comparisons, while no cellular component or molecular function GO terms were revealed (Fig. 2; Additional file 4: Table S3). The specific shoot down- and shoot_up-associated GO terms were related mainly to photosynthesis ('photosystem II assembly', 'photosynthesis, light reaction', 'chlorophyll biosynthetic process', 'chloroplast organization') and the stimulus response ('response to heat', 'cellular response to 


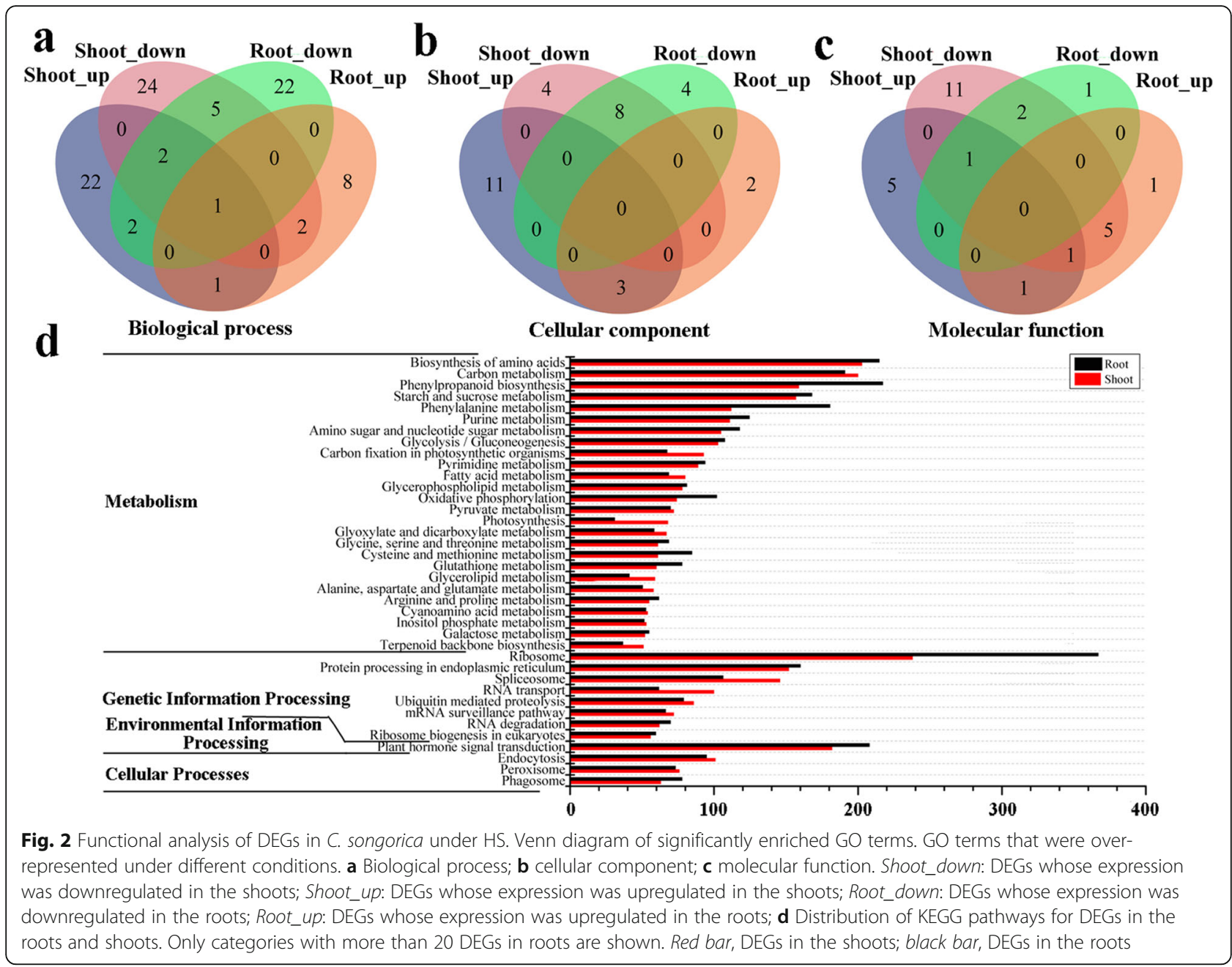

endogenous stimulus'), respectively, in the biological process category (Fig. 2a; Additional file 4: Table S3). Notably, root_down and root_up were associated mainly with morphogenesis ('cell morphogenesis', 'mitotic cell cycle process', 'developmental growth involved in morphogenesis') and phosphorylation ('phosphorylation', 'protein phosphorylation'), respectively (Fig. 2a; Additional file 4: Table S3). In the molecular function category, there were eight common GO terms between the shoot_down and root_down groups, which were related to many enzyme activities ('peroxidase activity', 'hydrolase activity'; Fig. 2c; Additional file 4: Table S3). In the cellular component category, the shoot_downspecific terms were similar to the biological process terms and were associated with the chloroplast ('chloroplast thylakoid', 'chloroplast'). The shoot_down and root_up groups presented five common terms, which were also related to the chloroplast ('chloroplast thylakoid membrane', 'chloroplast envelope', 'chloroplast stroma'; Fig. 2b; Additional file 4: Table S3).

\section{Key KEGG pathways of DEGs}

We investigated the key metabolic pathways that respond to HS in C. songorica. The results revealed several major metabolic pathways among the stress responses. The 'biosynthesis of amino acids' and 'ribosome' pathways were the most significant pathways involved in metabolism and genetic information processing, respectively (Fig. 2d). Interestingly, the numbers of DEGs associated with 'ribosome' significantly differed between the organs, with a 1.5 -fold greater number in the roots than in the shoots. Two pathways related to phenylpropanoids, 'phenylpropanoid biosynthesis' and 'phenylalanine metabolism', were significantly represented under HS and presented a greater number of DEGs in the roots than in the shoots. Furthermore, several DEGs were involved in energy metabolism, and their expression was downregulated under HS; these DEGs included those associated with the 'starch and sucrose', 'amino sugar and nucleotide sugar metabolism' and 'glycolysis/gluconeogenesis' categories (Fig. 2d). The expression of most of 
the DEGs involved in the photosynthesis pathway was also downregulated in C. songorica under HS, such as those in the 'carbon fixation in photosynthesis organisms' and 'photosynthesis' categories. With respect to genetic information processing, the expression of many DEGs related to 'RNA transport' and the 'spliceosome' was upregulated in C. songorica. In addition, two pathways presented more DEGs in the shoots than in the roots. DEGs in several other metabolic and signal transduction pathways, such as 'arginine and proline metabolism', 'pyrimidine metabolism', 'fatty acid metabolism' and 'plant hormone signal transduction' pathways, were also found to be differentially expressed in C. songorica (Fig. 2d).

\section{Differential expression of TF-encoding genes}

We also identified DEGs encoding members of TF families in C. songorica. In total, the genes encoding 1692 TFs belonging to 50 families were differentially expressed under HS in C. songorica (Additional file 5: Table S4). In the shoots and roots, 1692 and 1517 TFs, respectively, were identified among the DEGs. Strikingly, the number of downregulated TF genes was greater than the number of upregulated TF genes in the shoots and roots (Additional file 1: Fig. S3). In subgenome A and subgenome $\mathrm{B}$ of $\mathrm{C}$. songorica, the distribution of regulated TF genes was similar. Furthermore, we found that the numbers of upregulated and downregulated genes that encode TFs were greater in subgenome B than in subgenome $\mathrm{A}$ in the roots. The number of downregulated $\mathrm{TF}$ genes in subgenome $\mathrm{B}$ was greater than that in subgenome A only in the shoots (Additional file 1: Fig. $\mathrm{S} 3 \mathrm{~b})$. The greatest number of TFs belonged to the Ninlike family, followed by the MYB and NAC families (Table 1; Additional file 1: Fig. S3c). Furthermore, the numbers of several TF families significantly differed between the shoots and roots. The numbers of DEGs in the Nin-like, MYB and C3H families were greater in the shoots than in the roots (Table 1; Additional file 1: Fig. S3c). The number of DEGs encoding bHLH family members was lower in the shoots than in the roots. We observed that $23 \mathrm{HSF}$ members in $C$. songorica were homologous to 17 HSF TFs in rice. Furthermore, seven NF-YAs and seven NF-YBs TFs were identified in DEGs, respectively (Additional file 5: Table S4). The numbers of DEGs of several families, such as the NAC, ERF, bZIP, and WRKY families, were the same between the shoots and roots (Table 1). Overall, these results suggested that the regulation of TFs played a crucial role in the response of $C$. songorica to HS.

\section{Identification of a core set of Poaceae genes that are differentially regulated under HS}

To identify the functionally conserved genes in different species, we used four Poaceae species to identify the
Table 1 Distribution of transcription factors responsive to heat stress in C. songorica

\begin{tabular}{lll}
\hline $\begin{array}{l}\text { Transcription } \\
\text { factors family }\end{array}$ & $\begin{array}{l}\text { Transcription factors } \\
\text { in C.songorica shoot } \\
\text { DEGs }\end{array}$ & $\begin{array}{l}\text { Transcription } \\
\text { factors in C. } \\
\text { songorica root DEGs }\end{array}$ \\
\hline Nin-like & 169 & 121 \\
MYB & 157 & 126 \\
NAC & 117 & 120 \\
ERF & 106 & 105 \\
bHLH & 105 & 122 \\
C2H2 & 98 & 81 \\
C3H & 84 & 58 \\
bZIP & 82 & 77 \\
B3 & 78 & 78 \\
WRKY & 64 & 68 \\
FAR1 & 61 & 57 \\
G2-like & 59 & 43 \\
ARF & 50 & 47 \\
HD-ZIP & 48 & 36 \\
NF-YC & 36 & 36 \\
CO-like & 32 & 20 \\
Trihelix & 29 & 24 \\
GRAS & 27 & 27 \\
HSF & 23 & 19 \\
\hline Not Only categoies & mint & 27
\end{tabular}

Note: Only categories with more than 20 DEGs identified as transcription factors are shown

subsets of HS-responsive DEGs. In addition to these heat-responsive DEGs, we identified 1120, 888 and 2662 orthologues from rice, Hordeum vulgare and B. distachyon, respectively (Fig. 3a; Additional file 6: Table S5). In total, 216 orthologues that played a key role in the response to HS during evolution were identified in all the studied species (Fig. 3a; Additional file 6: Table S5). The GO enrichment analysis revealed that the conserved DEGs were enriched in 'stress responses' and 'photosynthesis'. For example, 37 and 41 conserved genes were enriched in the 'response to heat' and 'response to temperature stimulus', respectively. Furthermore, 12 conserved DEGs were involved in 'heat acclimation', and another 12 were involved in the 'response to osmotic stress' (Fig. 3b). Several other conserved DEGs were associated with chloroplasts; for example, 16 and 13 conserved DEGs were enriched in the 'chloroplast envelope' and 'chloroplast stroma', respectively. According to the prediction of the gene families via the Pfam database, a total of 31 gene families were identified. The largest family was the HSP family (15.3\%), followed by the leucinerich repeat receptor-like kinases (RLK) protein family $(10.6 \%)$, the subtilase family $(9.7 \%)$, the protein tyrosine kinase (PTK) superfamily (6.5\%), the phospholipase D 


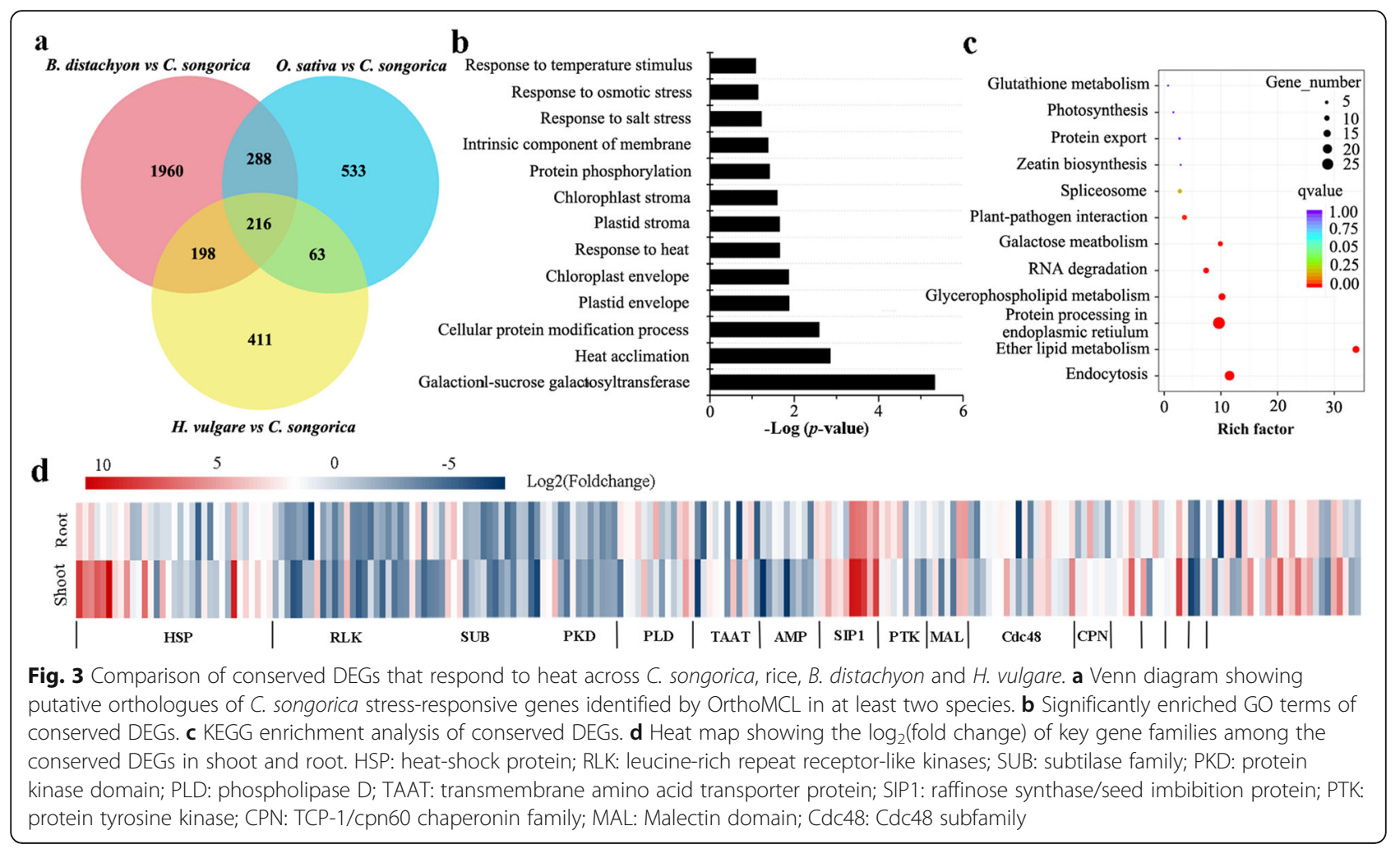

family (PLD, 6\%), and the raffinose synthase/seed imbibition protein family (SIP1, 4.6\%; Fig. 3d).

The KEGG pathway analysis revealed that some conserved DEGs were involved in the biosynthesis of different metabolites, including lipids and sugars (Fig. 3c). The conserved DEGs were most significantly enriched in 'ether lipid metabolism'. The expression of 12 and 2 putative $P L D$ genes was upregulated and downregulated in C. songorica, respectively (Additional file 6: Table S5). In terms of sugar metabolism, 10 conserved DEGs were annotated as raffinose synthase/seed imbibition protein, and the expression of these was upregulated in the shoots and roots. In addition to glutathione metabolism, the expression of glutathione s-transferase (GST) was downregulated in C. songorica. Remarkably, 'protein processing in endoplasmic reticulum' included 7 HSP genes whose expression was upregulated in $C$. songorica under HS. Moreover, among the conserved DEGs, the photosystem II subunit $R$ (PsbR) gene was identified, and its expression was upregulated (Additional file 6: Table S5).

KOG function classification indicated that these conserved DEGs involved in 'postertranslational medication, protein turnover, chaperones', 'signal transduction mechanisms' and lipid transport and metabolism' (Additional file 1: Fig. S4). The genes of RLK family were identified in the class of 'signal transduction mechanisms', most of which were downregulated in shoot and root under HS.

\section{Analysis of gene co-expression}

In order to measure the result of conserved responsive genes and identify the hub genes under HS, the coexpression network analysis was performed using the WGCNA package. The DEGs with low abundance $(\mathrm{FPKM} \leq 1)$ and low variability were filtered out in order to reduce noise. As a result of weighted co-expression network analysis, a module that was positively correlated with HS was identified and included 297 DEGs (Additional file 7: Table S6). The result of GO annotation showed that co-expressed DEGs involved stress responses (eg. 'response to stress' (sixty two DEGs), 'response to abiotic stimulus' (thirty five DEGs), 'response to temperature stimulus' (sixteen DEGs), 'response to heat' (six DEGs)), signalling (eg. 'signaling' (twenty one DEGs), 'signal transduction' (twenty one DEGs), 'hormone-mediated signaling pathway' (fourteen DEGs)) and regulations (eg. 'regulation of biosynthetic process' (thirty six DEGs), 'regulation of gene expression' (thirty four DEGs), 'regulation of response to stimulus' (nine DEGs) (Additional file 8: Table S7). Co-expressed DEGs were enriched in 'plant hormone signal transduction', 'plant-pathogen metabolism', and 'starch and sucrose metabolism' based on KEGG enrichment analyses (Additional file 1: Fig. S5).

In order to view the detailed expression patterns of pivotal individual genes, we assigned hub genes in coexpression network. The edge of co-expression network 
with low weight value (weight value $\leq 0.57$ ) were filtered out in order to reduce noise. Totally, 582 co-expression relationships and 152 DEGs were found in co-expression network under HS (Fig. 4, Additional file 9: Table S8). Based on the result of gene annotation, we found that the conserved heat-responsive DEGs of previous results were also identified in co-expression network, including six CsHSPs, five CsPTKs and four CsSIPs. This results suggested these conserved genes played a core role in regulatory networks of $C$. songorica under HS, and confirmed that our analysis results were reliable to reflect the regulatory networks of $C$. songorica under HS. In addition, we also found four TFs, including two CsNAC, one CsBZIP and one CsMYB genes (Fig. 4; Additional file 9: Table S8). In 'plant hormone signal transduction pathway', we found that one CsPP2C, four CsPTKs, one cold acclimation protein gene $(C s C O R)$ were significantly induced under HS. The one peroxisomal membrane protein (CsPEX), one $56 \mathrm{kDa}$ selenium binding protein (CsSBP56) and one sodium/calcium exchanger protein $(C s N C X)$ genes were annotated in 'inorganic ion transport and metabolism' pathway. In addition, two major intrinsic protein genes (CsMIP) belonged to aquaporin TIP2 and aquaporin SIP1, respectively (Fig. 4; Additional file 9: Table S8). Multiprotein bridging factor $1(C s M B F)$, a key regulated gene of HSFA1, were also identified in co-expression network. Other abiotic stress responsive genes were also identified in co-expression network, such as aldehyde dehydrogenase genes (CsALDH), Universal stress protein gene (CsUSP), RING- finger protein genes (CsRFP), Peroxidase (CsPOX), Catalase (CsCAT). Interestingly, some key hub genes belonged to unknown function family and didn't have gene annotation, such as CCG015511.1.gene, CCG015960.1.gene, CCG038756.1.gene, and CCG032589.1.gene (Fig. 4; Additional file 9: Table S8).

\section{Identification and analysis of HSP and HSF superfamily members in C. songorica}

Our results suggested that the HSPs and HSFs played core roles in HS response of $C$. songorica. Therefore, the

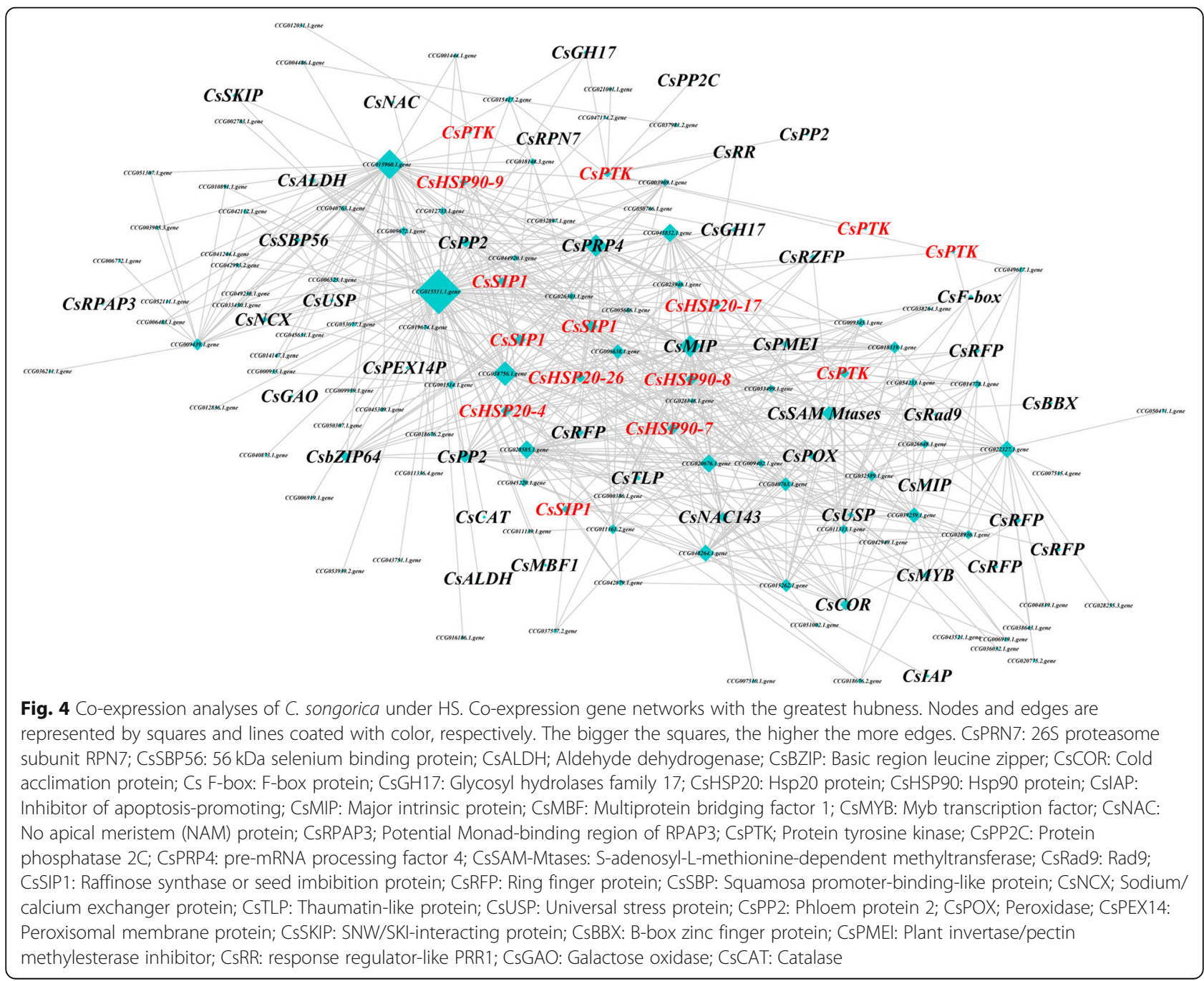


$H S P$ and $H S F$ superfamily of $C$. songorica were identified and analysed. Totals of 48, 25, 11, 20, 6 and 6 genes in the $C$. songorica genome were confirmed to be HSP2O, HSP70, HSP90, HSFA, HSFB, and HSFC genes, respectively, according to BLAST analysis (Fig. 5; Additional file 10: Table S9). These genes were named based on their chromosomal location and group. Analysis of the expression pattern revealed that 44 CsHSP2O (91.7\%), 22 CsHSP70 (88\%), 11 CsHSP90 (100\%), 20 CsHSFA (100\%), 5 CsHSFB (88.3\%) and 6 CsHSFC $(100 \%)$ genes were expressed under HS in the shoots (Fig. 5c); the same result was found in the roots. The numbers of upregulated and downregulated CSHSP7O genes were almost identical in the shoots and roots, but there were more upregulated CsHSP9O and CsHSFA genes than downregulated ones in these groups in the shoots and roots. Remarkably, there were more upregulated than downregulated $C s H S B$ genes in the shoots, but there were more upregulated than downregulated CSHSP2O and CsHSFC genes in these groups in the roots (Fig. 5c). The RT-qPCR results showed that HS significantly increased the expression levels of CsHSFA3-1, CsHSFA3-2,
CsHSFA9-1, CsHSFA9-2 and CsHSFA1-2 in C. songorica; however, the expression levels of CsHSP70-7 and CsHSP90-6 decreased under HS (Fig. 9b).

To analyse their evolutionary relationships, the HSP and HSF proteins of Arabidopsis, rice, B. distachyon and $C$. songorica were used to construct an unrooted phylogenetic tree. In total, 299 HSP proteins and 126 HSF proteins were included in the phylogenetic tree (Fig. 6; Additional file 1: Fig. S6). The phylogenetic branch of the HSP proteins was divided into the HSP20, HSP70 and HSP90 families (Fig. 5a). The 34 HSP90 proteins were classified into three subfamilies, which were designated as subfamilies I, II and III (Fig. 5). Subfamily I of HSP90 contained the most CsHSP90 proteins (6 CsHSP90s, 54.5\%). In addition, 104 HSP70 proteins were divided into 6 groups, designated as groups I, II, III, IV, $\mathrm{V}$ and VI. Among the six groups of HSP70 proteins, groups I and V were the largest (36 CsHSP70s, 34.6\%) and smallest (5 CsHSP70s, 4.8\%), respectively (Fig. 6). Notably, group III lacked HSP70 proteins from Arabidopsis. Moreover, the UAP XI, UAP X, and UAP VI subfamilies contained HSP20 proteins from only

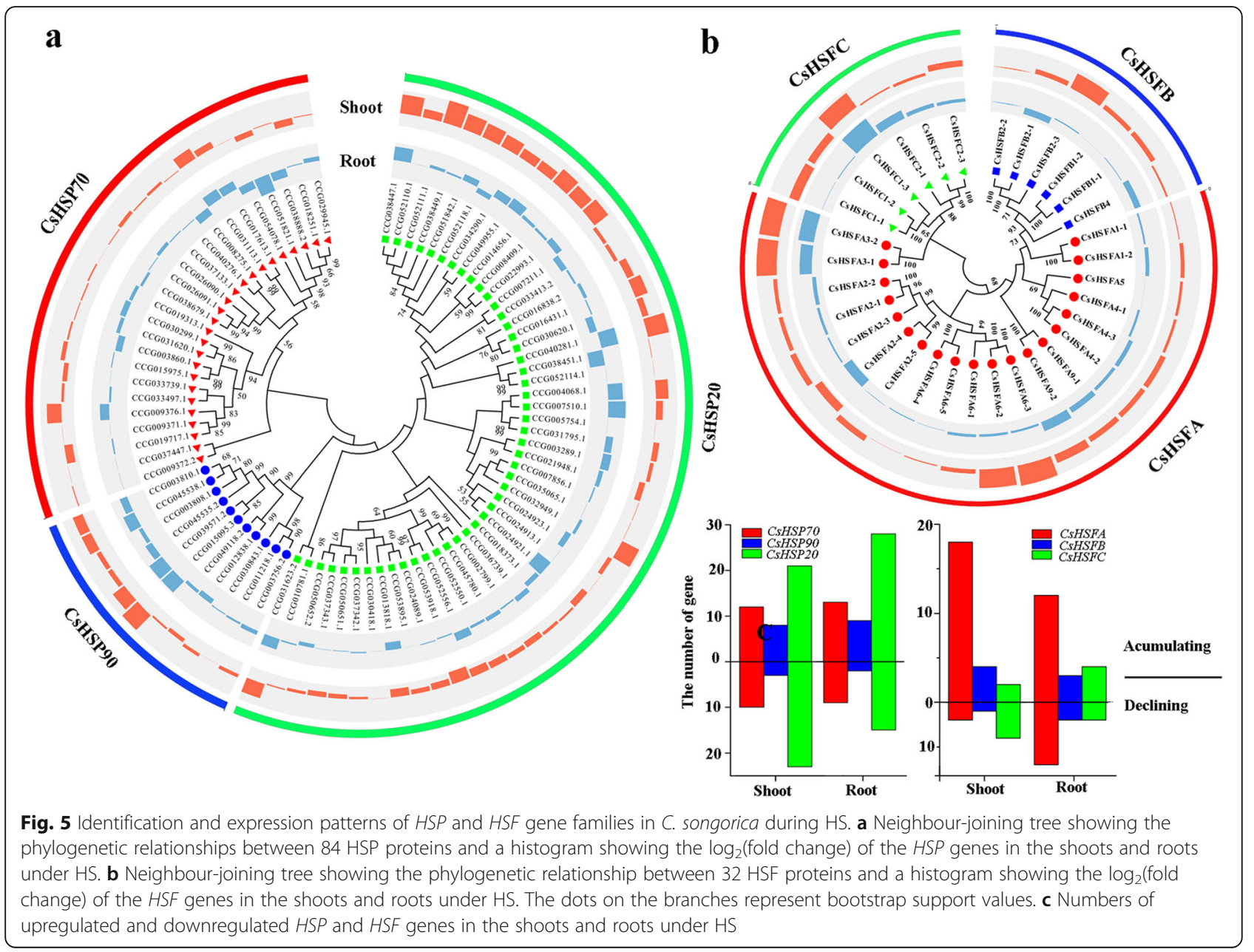




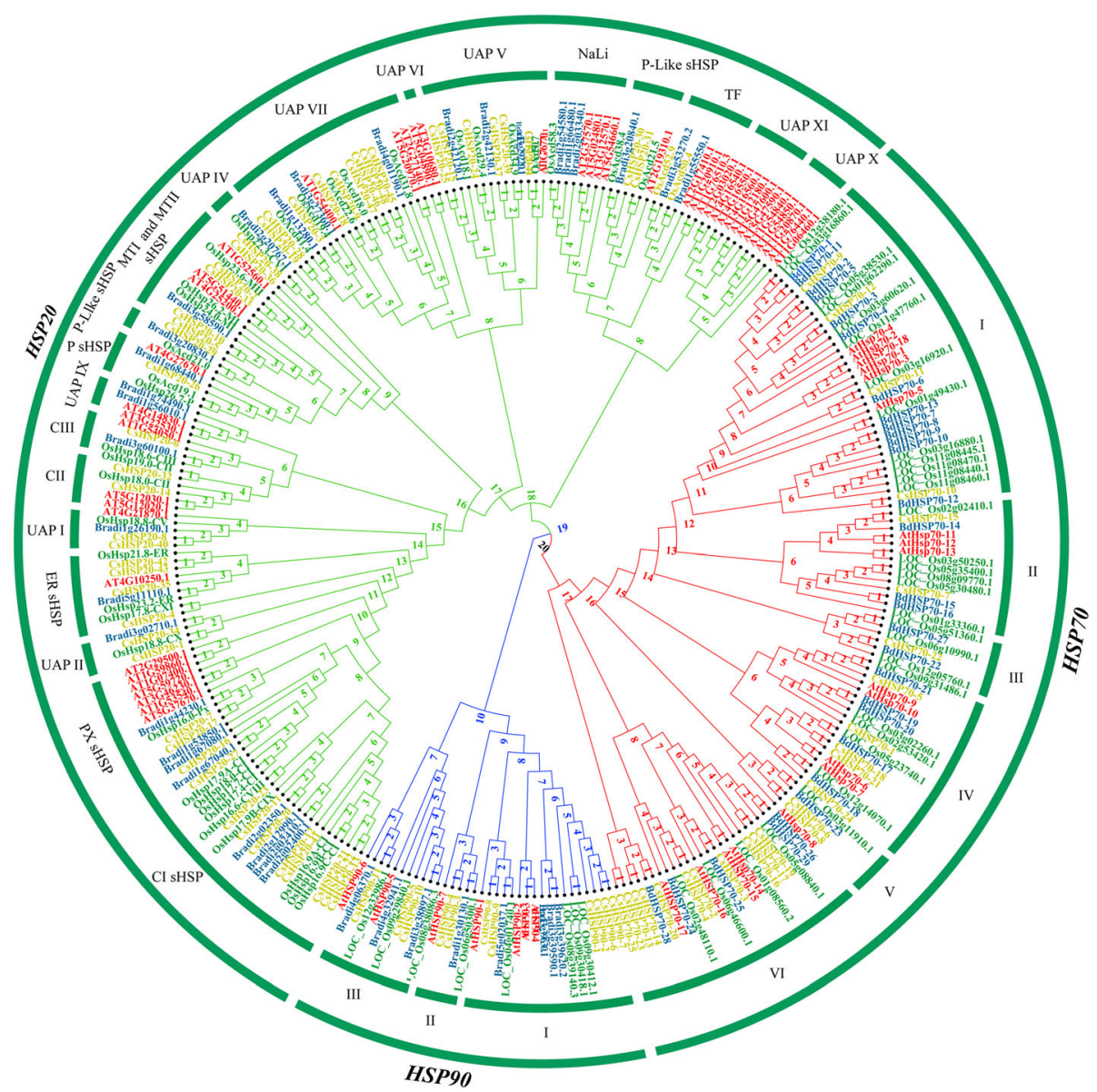

Fig. 6 Phylogenetic tree of HSP proteins in four species: C. songorica (yellow), rice (green), B. distachyon (blue) and Arabidopsis (red). The tree was generated with IQTREE software via the ML method

Arabidopsis, and the UAP V, UAP IV, UAP I and UAP II subfamilies were specific to Poaceae species (Fig. 6). The HSF proteins were divided into three subfamilies: HSFA, HSFB, and HSFC. The HSFA subfamily was the largest subfamily and contained nine groups (Fig. $5 \mathrm{~b}$ ). Notably, CsHSF was not grouped with HSFA7, HSFA8, or HSFB3 (Additional file 1: Fig. S6).

\section{Genome synteny and variation analysis of the HSP and HSF families in C. songorica}

Totals of 83 CsHSP (98.8\%) and 30 CsHSF (93.8\%) genes were located on the chromosomes of $C$. songorica, and these genes were found to be randomly distributed (Fig. 7; Additional file 11: Table S10). These genes were also evenly distributed between the two subgenomes of C. songorica. CsB10, CsA01 and CsB07 contained the most CsHSP2O genes, whereas no CsHSP2O genes were found on CsB09, CsA12, or CsB18 (Fig. 7). However, there were more CsHSP7O genes within subgenome B (13 CsHSP genes) than within subgenome A (9 CsHSP genes). The CsHSP9O genes were located on only 6 chromosomes, among which the majority were distributed on CsB02 and CsA05. CsHSFC genes were located only on CsB02, CsA05 and CsA12, and eight chromosomes of $C$. songorica did not contain any CsHSFA genes (Additional file 1: Fig. S7). We then identified homologous gene pairs, and the results showed that 25 homologous gene pairs belonged to the CsHSP gene family, including 16, 2, and 3 homologous gene pairs between subgenome $\mathrm{A}$ and subgenome $\mathrm{B}$, within subgenome $\mathrm{A}$, and within subgenome B, respectively (Fig. 7). Totals of 15,4 and 2 homologous gene pairs were identified in the CsHSP20, CsHSP7O and CsHSP9O families, respectively. In addition, there were 13,2 and 2 homologous gene pairs in the CsHSP20, CsHSP70 and CsHSP90 families between subgenome A and subgenome $\mathrm{B}$. We also identified 24 homologous gene pairs in the CsHSF gene family, among which 14, 4 and 6 belonged to the CsHSFA, CsHSFB and CsHSFC groups, respectively (Additional file 1: Fig. S7). Most of the homologous gene pairs presented a $\mathrm{Ka} / \mathrm{Ks}$ ratio $<0.3$, with the highest ratio recorded for CsHSP2O-48-CsHSP2O-45 $(\mathrm{Ka} / \mathrm{Ks}$ ratio = 


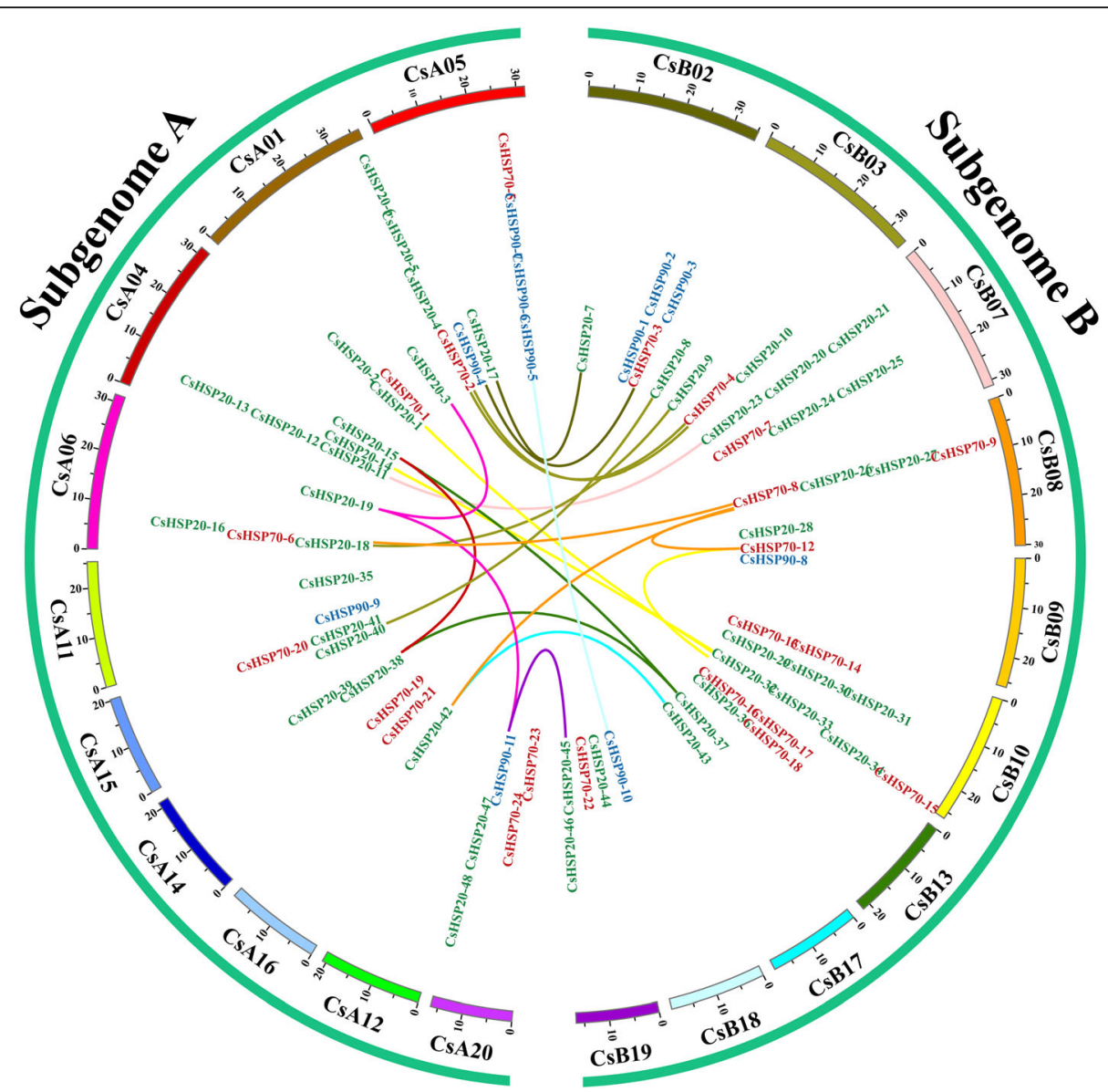

Fig. 7 Distribution and synteny analysis of C. songorica HSP genes. The 20 C. songorica chromosomes are shown as different coloured partial circles, and the chromosome numbers are indicated at the top of each bar. The coloured links indicate HSP syntenic regions in the C. songorica HSP gene family. HSP2O, green; HSP70, red; HSP90, blue

1.03). A similar trend was found for homologous gene pairs in the CsHSF gene family (Additional file 11: Table S10).

\section{Evolutionary and phylogenetic relationships among rice and C. songorica HSP and HSF genes}

To deduce the evolutionary history, origin, and function of the CsHSP and CsHSF genes effectively, we performed a comparative genomic analysis between rice and $C$. songorica. Rice is one of the best-studied model plant species and is closely phylogenetically related to C. songorica. A synteny map of the rice and $C$. songorica genomes was constructed, revealing 49 and 22 orthologous gene pairs between the $H S P$ and $H S F$ genes, respectively (Fig. 8; Additional file 1: Fig. S8; Additional file 12: Table S11). Among these genes, 34, 12, 3, 15, 4 and 3 orthologous gene pairs were identified from the HSP20, HSP70, HSP90, HSFA, HSFB and HSFC groups, respectively. Notably, 9, 7, 1, and 1 orthologous gene pairs (one to one) were identified in the HSP20, HSP70, HSP90, and $H S F B$ groups, respectively. We also found that one CsHSP and CsHSF gene each corresponded to multiple rice genes (Fig. 8; Additional file 1: Fig. S8). To explore the divergence of orthologous gene pairs between rice and $C$. songorica, the $\mathrm{Ka} / \mathrm{Ks}$ ratios of the orthologous gene pairs were calculated on the basis of the information within the comparative synteny map. Most of the orthologous gene pairs presented $\mathrm{Ka} / \mathrm{Ks}$ ratios $<0.3$, with the highest ratio presented by CsHSP2O-18-LOC_ Os03g06170.1 (Ka/Ks ratio $=0.48$; Additional file 12: Table S11). A similar trend was found for the homologous gene pairs of the CsHSF gene family.

\section{Discussion}

Heat stress is one of the key climatic parameters that affects plant growth and development. However, wholetranscriptome studies of $C$. songorica during HS are scarce. C. songorica is widely cultivated as ecological grass species in North-west China. To better understand the response of temperate grasses to HS, we measured both physiological parameters and large transcriptomic perturbations in heat-treated whole $C$. songorica seedlings for 0 to $72 \mathrm{~h}$. HS can limit photosynthesis, causing 


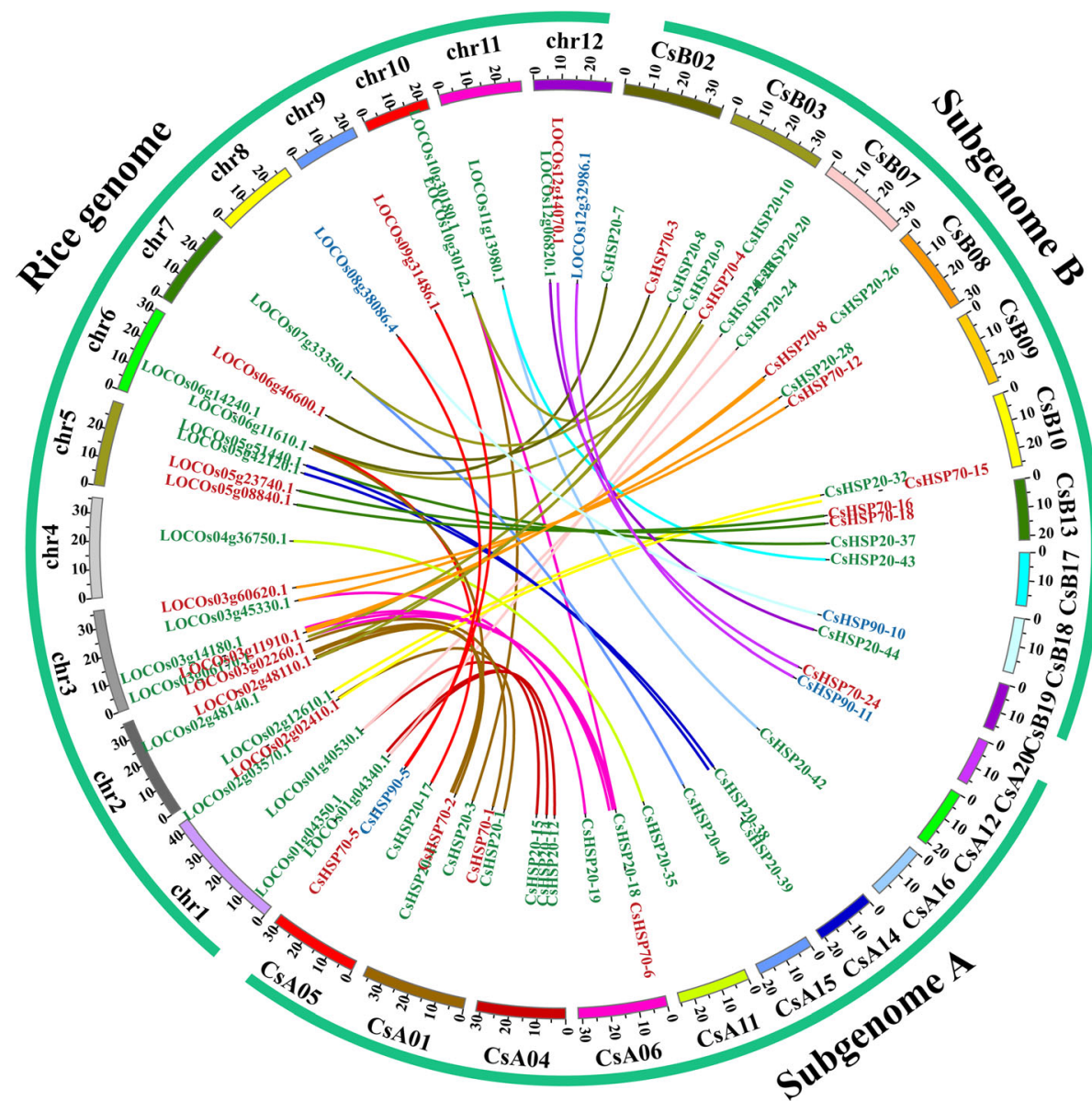

Fig. 8 Distribution and synteny analysis of HSP genes between C. songorica and rice. The coloured links indicate HSP syntenic regions in the C. songorica and rice HSP gene families. Chr1 to Chr12 belong to rice, whereas subgenome A and subgenome B represent the 20 chromosomes of C. songorica

a significantly decrease in the RWC. The effects of HS on plant photosynthesis can be evaluated by measuring chlorophyll fluorescence [31]. In C. songorica, the Fv/ Fm, Y(II) and rETRmax values significantly decreased in response to $6 \mathrm{~h}$ of $\mathrm{HS}$, indicating that C. songorica photosynthesis was limited by rETRmax under those conditions (Additional file 1: Fig. S1). We found that most of the genes whose expression was downregulated were involved in the chloroplast and its structural components, including multiple cellular components, which included GO terms such as 'chloroplast stroma', 'chloroplast envelope', 'chloroplast thylakoid membrane' and 'chloroplast thylakoid'. In the biological process category, most of the genes whose expression was downregulated were related to photosynthesis and chlorophyll, including GO terms such as 'photosystem II assembly', 'chlorophyll biosynthetic process', 'photosynthesis, light reaction' and 'chloroplast organization' (Fig. 2). These results indicated that decreases in photosynthesis pathways may contribute to improved heat resistance in $C$. songorica. It is widely accepted that Pro acts as a cellular osmolyte and can increase plant resistance by accelerating biosynthesis [32]. In addition, Pro participates in the maintenance of redox balance and the scavenging of reactive oxygen species (ROS) [33]. Notably, 'proline metabolism' was identified among the enriched KEGG pathways of the DEGs. The expression of the associated genes was upregulated in C. songorica roots and shoots under HS. In addition, the Pro content was significantly greater in C. songorica under HS than under control conditions. These results indicated that Pro plays a positive role in the $C$. songorica response to HS, which has been confirmed in various plant species, such as kentucky bluegrass and cotton $[34,35]$.

Because C. songorica is an allotetraploid plant species, we identified the distribution of DEGs between its two subgenomes. Interestingly, the DEGs were evenly distributed between the two subgenomes. Specifically, 6971 and 7172 DEGs were distributed within subgenome A and subgenome B, respectively (Fig. 1c). We also found 
that the number of upregulated and downregulated genes did not differ between the two subgenomes. For instance, 4255 and 4298 DEGs whose expression was downregulated were identified in subgenome A and subgenome B, respectively, in C. songorica shoots. These results suggested that the two subgenomes of $C$. songorica played an equal role in the response to HS.

TFs are among the most promising targets for the improvement of plant performance under abiotic stress [36]. Members of certain TF families, such as the HSF, NF-YA, NF-YB, NF-YC and bZIP families, are known to mediate the HS response in plants [37-40]. In the present study, the expression of 1692 TFs from 50 families was found to be regulated in $C$. songorica in response to HS (Table 1). In total, 23 HSF, 7 NF-YA, 7 NF-YB and 36 NF-YC TFs were identified in C. songorica. HSFA1 has been predicted to directly regulate the expression levels of important heat-responsive TFs, such as DREB2A, HSFA2, HSFA7 and HSFBs [18]. Here, we found that one, two and one putative $H S F$ genes from $C$. songorica were homologous to the rice HSFA1, HSFA2 and HSFA9 genes, respectively.

Comparisons of transcriptomes between different species can provide information about the conservation of gene functions throughout evolution. In total, 216 orthologues were identified in all species; these genes constituted a core set of evolutionarily conserved genes associated with HS (Fig. 3). These conserved genes were largely involved in stress responses and activities within chloroplasts. KEGG pathway analysis revealed that the conserved genes were involved in 'glutathione metabolism', 'endocytosis' and 'zeatin biosynthesis'. These pathways are known to be involved in responses to abiotic stress. Glutathione eliminates free radicals and protects the plant body [41]. Moreover, conserved genes that were associated with 'glutathione metabolism' and whose expression was downregulated under HS, which were homologous with the GST gene in Arabidopsis. Previous studies have shown that AtGSTU17 was involved in glutathione metabolism under abiotic stress. When AtGSTU17 was mutated, plants were more tolerant to drought and salt stresses compared with wild-type plants [42]. Endocytosis plays a core role in maintaining cell homeostasis, developmental processes, programmed cell death and stress tolerance $[43,44]$. In the present study, 13 PLD genes involved in endocytosis and endosomal recycling pathways were identified. Furthermore, PLDs are involved in abscisic acid (ABA) signalling, drought, cold and salinity responses $[45,46]$. Members of the $L R R-R L K$ family, which constitutes one of the largest gene families, are involved in pathogen responses, development and abiotic stress responses in plants [46, 47]. In addition, RLKs and $\mathrm{Ca}^{2+}$ channels act as membrane-anchored proteins could sense the increasing membranes fluidity under HS (Fig. 9a) [48]. In total, we identified 23 conserved $L R R-R L K$ genes within $C$. songor$i c a$. Expression of nineteen of these $23 L R R-R L K$ genes was downregulated in the shoots and roots under HS.

Based on the results of co-expression, some stress response genes were identified, which involved in posttranslational modification, signal transduction mechanisms, defense mechanisms, energy production and conversion, and inorganic ion transport and metabolism (Fig. 4). In model plants, HSP and HSF genes play a central role in HS and acquired thermotolerance [49]. $H S P$ and HSF genes are also essential for normal growth and development in plants. Notably, the largest families of conserved DEGs identified belonged to the HSP superfamily, including 6 HSP20, 18 HSP70 and 9 HSP9O genes (Fig. 3). The HSPS were also identified to overlap genes of conserved genes and co-expression genes. We suggested HSPS play a core role in regulatory networks of $C$. songorica under HS, and confirmed our analysis results were reliable to reflect the regulatory networks of C. songorica under HS. Therefore, we identified the HSP and HSF superfamily members in the $C$. songorica genome. There are 84 CsHSP and 32 CsHSF genes in the C. songorica genome, including 48 HSP2O, 25 HSP70 and 11 HSP9O genes (Fig. 5). We also found that $72.7 \%$ of the HSP genes were upregulated in $C$. songorica under HS. Compared with that which occurs in Arabidopsis, rice and $B$. distachyon, the results suggested that the HSP and HSF family of $C$. songorica has not tended to expand $[22,50,51]$, which was confirmed by the $\mathrm{Ka} / \mathrm{Ks}$ values of 21 homologous gene pairs of less than 0.3 in that species (Fig. 7; Additional file 1: Fig. S7). Furthermore, the CsHSP and CsHSF genes were evenly distributed between the two subgenomes of $C$. songorica. The $\mathrm{Ka} / \mathrm{Ks}$ ratios of the orthologous gene pairs between rice and $C$. songorica also indicated that rice and $C$. songorica might have undergone purifying selection during their long evolutionary history.

\section{Conclusions}

In this study, we performed conserved HS response genes identify, gene co-expression network analysis, and gene family identify by using RNA-seq data and genome information to reveal regulator network of $C$. songorica under HS. In this process, RLKs and $\mathrm{Ca}^{2+}$ involved in perceiving external signals in $C$. songorica under $\mathrm{HS}$. HSFA1 is first activated by the inhibition of HSP70/ HSP9O (Fig. 9a). The upregulation of CsHSFA1 in turn plays a key role in activating the expression of downstream TFs such as DREB2A, HSFA2 and HSFBs. HSFBs then inhibit the activity of $C S H S F A 1$ via a negative feedback loop (Fig. 9a). DREB2A regulates the expression of chaperones and enzymes in response to HS together with $N F-Y A 2, N F-Y B 3$, etc. Other pathways were also 


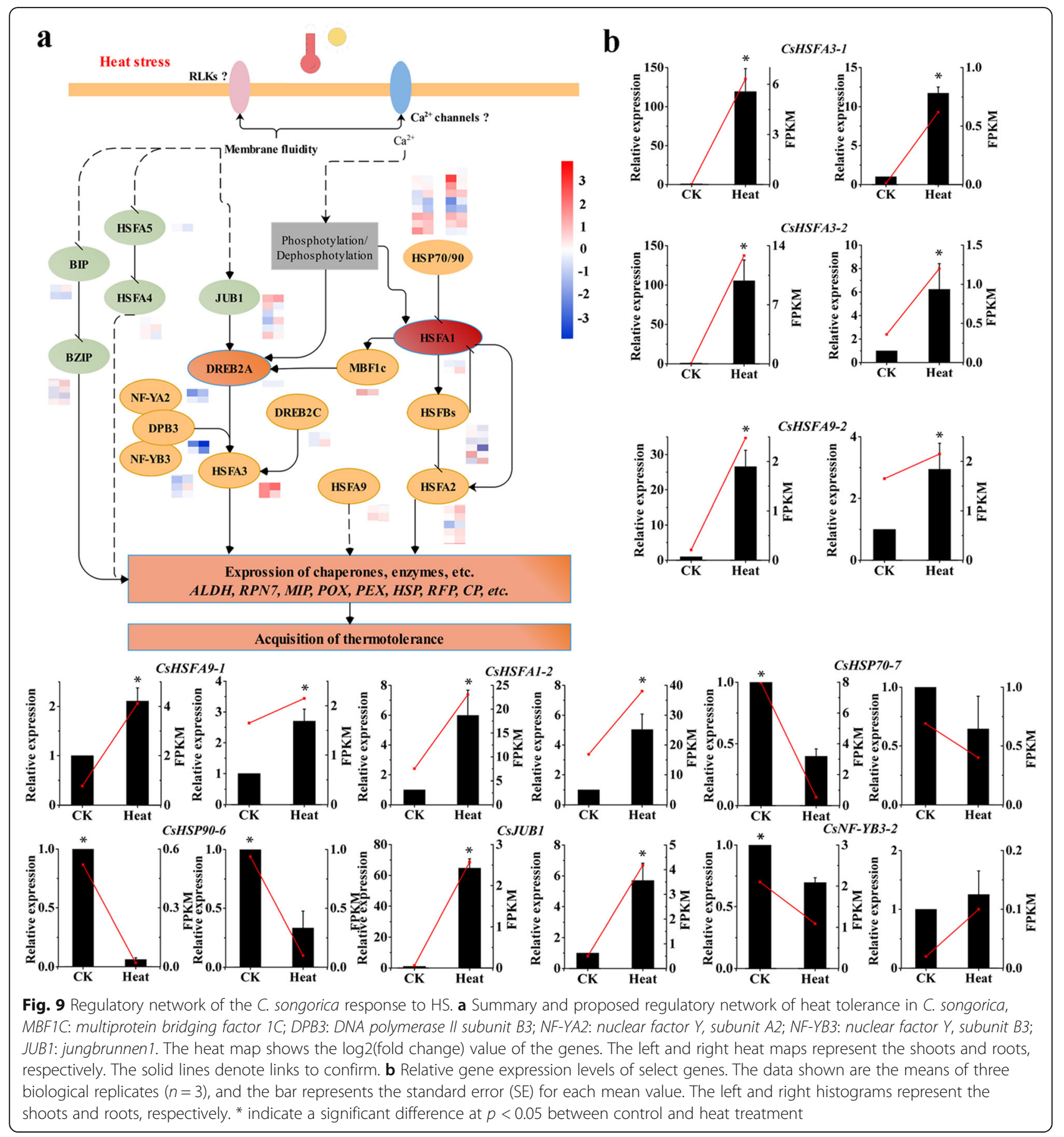

found to be important, including those involving HSFA5/HSFA4 and BIP (Fig. 9a). The transcriptional regulatory network ultimately affects the expression of enzymes and chaperones via high expression levels of CSHSFA3 and CsHSFA9, which improves expression of chaperones and enzymes, and the thermotolerance and adaptation of C. songorica (Fig. 9b). This study revealed the regulatory network of $C$. songorica under HS on the basis of a comprehensive transcriptomic analysis and the identification of conserved genes. Our results provide comprehensive data for dissecting the molecular mechanism of HS responses in C. songorica and provide clues for thermotolerance improvement in grasses.

\section{Materials and methods}

Plant material and experimental treatments

Seeds of $C$. songorica were obtained from the C. songorica production field of Lanzhou University $\left(103^{\circ} 08^{\prime} \mathrm{N}\right.$, 
$\left.38^{\circ} 62^{\prime} \mathrm{E}\right)$, Minqin County, Gansu Province, China. The seeds of $C$. songorica were sown in a sand:vermiculite (1: $1, \mathrm{v} / \mathrm{v})$ mixture. The plates were maintained in a growth chamber supplemented with $150 \mu \mathrm{mol}$ quanta $\mathrm{m}^{-2} \mathrm{~s}^{-1}$ irradiance under a photoperiod of $16 / 8 \mathrm{~h}$ (light/dark), $28 / 24{ }^{\circ} \mathrm{C}$ day/night temperatures, and $65 \%$ relative humidity [5]. Four-week-old seedlings were transplanted into plastic pots (height, $8 \mathrm{~cm}$; top diameter, $8 \mathrm{~cm}$; bottom diameter, $6 \mathrm{~cm}$ ), with two plants per pot. Each pot contained $450 \mathrm{~g}$ of a sand:vermiculite $(1: 1, \mathrm{v} / \mathrm{v})$ mixture. The experiment of temperature treatment was repeated in three growth chambers. After 5 weeks of cultivation, the temperature was increased to $40^{\circ} \mathrm{C}$ to impose $\mathrm{HS}$ for 3 days. The shoots and roots were harvested after $0 \mathrm{~h}, 6$ h, $12 \mathrm{~h}, 24 \mathrm{~h}, 36 \mathrm{~h}, 48 \mathrm{~h}$ and $72 \mathrm{~h}$ of HS, after which they were immediately frozen in liquid $\mathrm{N}_{2}$ and stored at $80{ }^{\circ} \mathrm{C}$ for physiological and transcriptomic analyses.

\section{Characterization of physiological and photosynthesis traits}

For the characterization of physiological and photosynthesis traits, eight seedlings were collected from the control group and the treatment group at the corresponding time points. The leaf materials of four seedlings were quickly harvested and used for the estimation of physiological traits, including MDA content, Pro content, and RWC. The MDA content and Pro content were determined according to the instructions provided by the manufacturer of the reagents (MDA-2-Y, PRO-1-Y; Komin, Suzhou, China; http://www.cominbio.com/). Afterward, the leaf samples were dried at $60{ }^{\circ} \mathrm{C}$ for $72 \mathrm{~h}$ to determine the RWC, which was calculated as follows: $\mathrm{RWC} \%=($ fresh weight-dry weight $) /$ (saturated weight-dry weight)*100 [52]. An Imaging-PAM instrument (Waltz $\mathrm{GmbH}$, Effeltrich, Germany) was used to measure Fv/ Fm, Y(II) and rETR $_{\max }$ according to the manufacturer's instructions [53]. Leaf temperature and soil temperature were measured with chlorophyll fluorescence system (Heinz Walz, Germany) and soil thermometer, respectively.

\section{Illumina RNA-Seq library construction and sequencing}

Total RNA from each shoot and root was isolated via TRIzol reagent (Invitrogen, USA) according to the manufacturer's instructions. Sequencing libraries were generated via a NEBNext ${ }^{\oplus}$ Ultra $^{\mathrm{Tm}}$ RNA Library Prep Kit for Illumina ${ }^{\circledR}$ (NEB, USA) following the manufacturer's recommendations. M-MuLV reverse transcriptase $\left(\mathrm{RNase} \mathrm{H}^{-}\right.$) and random hexamer primers were used to synthesize first-stand cDNA. Second-stand synthesis was subsequently performed via DNA Polymerase I and RNase H. A total of 12 libraries (2 temperature treatment $\times 2$ tissues $\times 3$ replicates) were sequenced on the Illumina HiSeq platform, and $125 \mathrm{bp}$ paired-end reads were generated. Low-quality reads, poly- $\mathrm{N}$ sequences from the raw data and reads containing adapters were removed to generate clean reads. Furthermore, HISAT2 software was used to map the clean reads to the $C$. songorica genome [4, 54], and StringTie (1.3.1) was used to quantify the gene expression levels according to the fragments per kilobase of transcript per million fragments (FPKM) method [55]. DEGs were analysed by the DESeq $\mathrm{R}$ package. The false discovery rate (FDR) was adjusted via the posterior probability of being differentially expressed (PPDE) method. Genes were considered differentially expressed when they presented a fold change $\geq 2$ and an FDR $\leq 0.01$. Three biological replicates were used for each sample. The GO annotation, GO enrichment and KEGG pathways were estimated via the BMK Cloud server (www.biocloud.net).

A heat map of gene expression was generated using OmicShare tools (http://www.omicshare.com/tools), and the Circos 0.69 program was used to show gene expression and gene locations on a genome-wide scale [56]. Last, a Venn diagram was generated by the jvenn website (http://jvenn.toulouse.inra.fr/app/example.html).

\section{Identification of TFs and conserved DEGs in response to HS in the Poaceae}

A TF analysis was performed by the BMK Cloud sever, an online platform for data analysis (http://www.biocloud.net/). To identify subsets of HS-related DEGs shared between $C$. songorica, rice, $H$. vulgare and $B$. distachyon [57], select SRA data related to HS were downloaded from the NCBI database (PRJNA530826; PRJNA360513; PRJNA324116). The data analysis methods were the same as those described previously. OrthoMCL software V5 was used to identify orthologues with the default settings [58], and gene families were identified by Pfam (https://pfam.sanger.ac.uk/; e-value cut-off $>1 \mathrm{e}^{-5}$ ).

\section{Gene co-expression network analysis}

The 59 RNA-seq data under abiotic stresses including data from this article were used to co-expression analysis [40]. According to the result from each sample regarding the DEGs expression quantity, we removed the expression quantity from all samples that were $<1$ and expressions that barely changed (DEGs variance was $<0.75$ ). $\mathrm{R}$ package WGCNA was used to construct gene co-expression networks [59]. The soft threshold (power) value with a signed $\mathrm{R}^{2}$ threshold $>0.85$ was analysed by using PickSoftThreshhold function. We then used the automatic network construction function blockwiseModules to obtain weighted co-expression clusters, called modules, with the following settings for the calculation processes: power = 14, networkType $=$ unsigned, corType $=$ pearson, minModuleSize $=30$, and mergeCutHeight $=0.25$. A module that 
was significantly $(p<0.01)$ related to the heat stress samples is selected was selected to analysis in this study. Co-expression network was showed using Cytoscape (http://cytoscapeweb.cytoscape.org/).

\section{Identification and analysis of HSP and HSF gene families in the $C$. songorica genome}

The protein and gene sequences of HSPs and HSFs derived from Arabidopsis, rice and B. distachyon were downloaded from Phytozome 12 (https://phytozome.jgi. doe.gov/pz/portal.html). These sequences served as queries for identifying the genes encoding the CsHSP and CsHSF proteins within the $C$. songorica genome via BLAST software (e-value cut-off $>1 \mathrm{e}^{-5}$ ). CD-HIT (http:// weizhongli-lab.org/cdhit_suite/cgi-bin/index.cgi) tools were subsequently used to remove redundant sequences. The conserved domains within the candidate CsHSP20, CsHSP70, CsHSP90 and CsHSF proteins were further examined via Pfam (https://pfam.sanger.ac.uk/; e-value cut-off $>1 \mathrm{e}^{-5}$; PF00011; PF00012; PF02518; PF00447). Multiple sequence alignment of the HSP and HSF proteins of C. songorica was performed with ClustalX, with the default parameters. An evolutionary tree of the CsHSP and CsHSF proteins was then constructed with MEGA 7 software, with 1000 bootstrap replicates (http://www.megasoftware.net).

The HSP and HSF proteins of C. songorica, Arabidopsis, rice and $B$. distachyon were used for phylogenetic analysis (Additional file 13: Table S12). As such, their sequences were aligned with ClustalX software, with the default parameters. A maximum likelihood (ML)-based phylogenetic tree was constructed via IQTREE (1.6.11 version) software with 1000 bootstrap replicates [60]. The Shimodaira-Hasegawa-like approximate likelihood ratio test (SH-alRT branch test) was used to test the branch support, and the consensus tree topology was visualized with FigTree (version 1.4.3).

\section{Synteny analysis and chromosomal location of HSP and HSF genes}

We used MCScanX software to search for gene pairs within the $C$. songorica and between the $C$. songorica and rice genomes (BLASTP: $\mathrm{E}<1 \mathrm{e}-5$, top 3 matches) [61]. A synteny map was generated via the Circos 0.69 program. The synonymous (Ks) and nonsynonymous (Ka) nucleotide substitutions were determined via ClustalW, PAL2NAL and the bio-pipeline yn00 program (https://github.com/tanghaibao/bio-pipeline/tree/master/ synteny-pipeline).

\section{Quantitative real-time (RT) PCR}

Total RNA was isolated from C. songorica shoots and roots after stress treatments via RNAiso reagent (TaKaRa, Dalian, China). For reverse transcription, first- strand cDNA was synthesized by a FastKing RT Kit (with gDNase; Tiangen, China). 2X TaqMan Fast qPCR Master Mix (Sangon, China) and a CFX96 instrument (Bio-Rad) were used with default settings to calculate the threshold cycle (CT) values. CsGAPDH was used as a reference gene. The specific primers used for the selected genes are shown in Additional file 14: Table S13. The relative expression levels were calculated by the comparative CT method [62]. All reactions were performed in triplicate.

\section{Supplementary information}

Supplementary information accompanies this paper at https://doi.org/10. 1186/s12864-020-07122-8.

Additional file 1: Figure S1. Effects of HS on the phenotype and physiological traits of $C$. songorica seedlings. (a) Phenotype of $C$. songorica at different time points under heat treatment. (b)-(g) Leaf RWC, MDA content, Pro content, Fv/Fm, rETRmax and quantum yield II. (h)-(i) Leaf temperature and soil temperature. Bars with different letters indicate significant differences at $P \leq 0.05$ (Duncan's test). Figure S2. GO enrichment of DEGs in the shoots and shoots of $C$. songorica under HS. Red bar, DEGs in the shoots; green bar, DEGs in the roots. Figure S3. Summary of identified TFs encoded by DEGs in C. songorica upon HS. Figure S4. KOG function classification of conserved DEGs; Figure S5. KEGG pathway enrichment of co-expression genes; Figure S6. Phylogenetic tree of HSF proteins in five species: C. songorica (yellow), rice (green), B. distachyon (blue), Z. mays (purple) and Arabidopsis (red). The tree was generated with IQTREE software via the ML method; Figure S7. Distribution and synteny analysis of C. songorica HSF genes. In the Fig., the 20 C. songorica chromosomes are shown as different coloured partial circles, and the chromosome numbers are indicated at the top of each bar. The coloured links indicate HSF syntenic regions in the C. songorica HSF gene family; Figure S8. Distribution and synteny analysis of HSF genes between C. songorica and rice. In the Fig., the 20 C. songorica chromosomes are shown as different coloured partial circles, and the chromosome numbers are indicated at the top of each bar. The coloured links indicate HSP syntenic regions in the C. songorica and rice HSF gene families. Chr 1 to Chr12 belong to rice, whereas subgenome $A$ and subgenome $B$ represent the 20 chromosomes of $C$. songorica;

Additional file 2: Table S1. RNA-Seq data for twelve samples Additional file 3: Table S2. Significant DEGs (FDR $\leq 0.01$ ) were identified in at least one stress treatment, and their expression was regulated in the shoots and roots

Additional file 4: Table S3. List of over-represented significantly enriched GO terms shared between different combinations of organ/condition comparisons corresponding to the Venn diagram in Fig. 2a, b, c

Additional file 5: Table S4. Summary of DEGs that encode TFs identified in the transcriptome of $C$. songorica under HS

Additional file 6: Table S5. List of conserved heat-responsive genes shared across 4 Poaceae species and their expression patterns in $C$. songorica

Additional file 7: Table S6. The node and edge of co-expression network in C. songorica under HS

Additional file 8: Table S7. GO enrichment terms of co-expression genes of $C$. songorica under HS

Additional file 9: Table S8. The annotation and regulation of selected co-expression genes in C. songorica under HS

Additional file 10: Table S9. Characteristics of HSP and HSF genes in C. songorica;

Additional file 11: Table S10. Synteny blocks and $\mathrm{Ka} / \mathrm{Ks}$ values of HSP and HSF genes within the C. songorica genome 
Additional file 12: Table S11. Synteny blocks and $\mathrm{Ka} / \mathrm{Ks}$ values of HSP and HSF genes between the C. songorica genome and the rice genome Additional file 13: Table S12. The protein sequences of HSP and HSF from Arabidopsis, rice and B. distachyon and C. songorica

Additional file 14: Table S13. List of gene-specific primers used in this study.

\section{Abbreviation}

HS: Heat stress; HSP: Heat-shock protein; HSF: Heat-shock transcription factor; DEGs: Differentially expressed genes; TFs: Transcription factors; GO: Gene ontology; RT-qPCR: Real-time quantitative PCR

\section{Acknowledgements}

We thank the reviewers for their reading time and constructive comments.

\section{Authors' contributions}

QY, YW and JZ designed the study. FW and $J L$ participated in the data analysis. $Y Z$ and TM prepared the plant materials and performed the heat treatment. XZ, QM and PW performed the RT-qPCR experiments. QY performed most of the experiments, analysed the biological information and wrote the article, and JZ modified the language in the manuscript. All authors read and approved the final manuscript.

\section{Funding}

This work was financially supported by grants from the National Natural Science Foundation of China (31572453), the Science and Technology Program of Gansu Province (No. 19ZD2NA002), the Intellectual Property Right Program of Gansu Province (No. 19ZSCQ044), and the Open Project Program of Arid Meteorology (IAM201703). All of this funding plays roles in the design of the study and collection, analysis, and writing of the manuscript.

\section{Availability of data and materials}

The datasets generated and during the current study are publicly available in the Sequence Read Archive (SRA) at NCBI (SRP218434; PRJNA530826; PRJNA360513; PRJNA324116) repository. The C. songorica genome are publicly available in the in the National Genomics Data Center (https://bigd big.ac.cn/), under accession number GWHANUQ00000000 that is publicly accessible at https://bigd.big.ac.cn/gwh. The protein sequences of HSPs and HSFs derived from Arabidopsis, rice and B. distachyon were deposited in Additional file 14: Table S12.

\section{Ethics approval and consent to participate} Not applicable.

\section{Consent for publication}

Not applicable.

\section{Competing interests}

The authors declare that they have no competing interests.

Received: 31 May 2020 Accepted: 5 October 2020

Published online: 16 October 2020

\section{References}

1. Wahid A, Gelani S, Ashraf M, Foolad MR. Heat tolerance in plants: An overview. Environ Exp Bot. 2007;61(3):199-223.

2. Sillmann J, Kharin W, Zhang X, Zwiers FW, Bronaugh D. Climate extremes indices in the CMIP5 multimodel ensemble: Part 2.;Future climate projections. J Geophysical Res Atmospheres. 2013;118(6):2473-93.

3. Al JE. Paasche: IPCC [Intergovernmental Panel on Climate Change]. In: Climate Change 2007: The Physical Science Basis. Contribution of Working Group I to the Fourth Assessment Report of the IPCC. Cambridge, United Kingdom: Cambridge University Press; 2007.

4. Zhang J, Wu F, Yan Q, John UP, Cao M, Xu P, Zhang Z, Ma T, Zong X, Li J, et al. The genome of Cleistogenes songorica provides a blueprint for functional dissection of dimorphic flower differentiation and drought adaptability. Plant Biotechnol J. 2020. https://doi.org/10.1111/pbi.13483.

5. Yan Q, Wu F, Yan Z, Li J, Ma T, Zhang Y, Zhao Y, Wang Y, Zhang J. Differential co-expression networks of long non-coding RNAs and mRNAs in
Cleistogenes songorica under water stress and during recovery. BMC Plant Biol. 2019;19(1):23.

6. Zhang J, Duan Z, Zhang D, Zhang J, Di H, Wu F, Wang Y. Cotransforming bar and CSLEA enhanced tolerance to drought and salt stress in transgenic alfalfa (Medicago sativa L.). Biochem Biophys Res Commun. 2016;472(1):75-82.

7. Zhang J, Kong L, Liu Z, Jahufer Z, Duan Z, Huo Y, Di H, Wang Y. Stressinduced expression in Arabidopsis with a Dehydrin LEA protein from Cleistogenes songorica, a xerophytic desert grass. Plant Omics. 2015;8(6): 485-92.

8. Zhang J, Zhen D, Jahufer Z, An SJ, Wang YR. Stress-inducible expression of a Cleistogenes songorica ALDH gene enhanced drought tolerance in transgenic Arabidopsis thaliana. Plant Omics. 2014;7(6):438-44.

9. Muvunyi BP, Yan Q, Wu F, Min X, Yan ZZ, Kanzana G, Wang Y, Zhang J. Mining late embryogenesis abundant (LEA) family genes in Cleistogenes songorica, a xerophyte perennial desert plant. Int J Mol Sci. 2018;19(11): 3430

10. Zhu Y, Wang Z, Jing YJ, Wang LL, Liu X, Liu YX, Deng X. Ectopic overexpression of BhHsf1, a heat shock factor from the resurrection plant Boea hygrometrica, leads to increased thermotolerance and retarded growth in transgenic Arabidopsis and tobacco. Plant Mol Biol. 2009:71(4-5):451-67.

11. Bao AK, Wang YW, Xi JJ, Liu C, Zhang JL, Wang SM. Co-expression of xerophyte Zygophyllum xanthoxylum ZXNHX and ZXVP1-1 enhances salt and drought tolerance in transgenic Lotus corniculatus by increasing cations accumulation. Funct Plant Biol. 2014;41(2):203-14.

12. Mittler R, Finka A, Goloubinoff P. How do plants feel the heat? Trends Biochem Sci. 2012;37(3):118-25.

13. Zeller G, Henz SR, Widmer CK, Sachsenberg T, Laubinger S. Stress-induced changes in the Arabidopsis thaliana transcriptome analyzed using wholegenome tiling arrays. Plant J. 2009;58(6):1068-82.

14. Liu G, Zha Z, Cai H, Qin D, Jia H, Liu C, Qiu D, Zhang Z, Wan Z, Yang Y, et al. Dynamic transcriptome analysis of anther response to heat stress during anthesis in thermotolerant rice (Oryza sativa L.). Int J Mol Sci. 2020;21(3): 1155

15. Wang J, Lv J, Liu Z, Liu Y, Song J, Ma Y, Ou L, Zhang X, Liang C, Wang F, et al. Integration of transcriptomics and metabolomics for pepper (Capsicum annuum L.) in response to heat stress. Int J Mol Sci. 2019;20(20): 5042

16. Elzanati $\mathrm{O}$, Mouzeyar S, Roche J. Dynamics of the transcriptome response to heat in the Moss, Physcomitrella patens. Int J Mol Sci. 2020;21(4):1512.

17. Yi $X$, Bingru $H$. Transcriptomic analysis reveals unique molecular factors for lipid hydrolysis, secondary cell-walls and oxidative protection associated with thermotolerance in perennial grass. BMC Genomics. 2018;19(1):70.

18. Ohama N, Sato H, Shinozaki K, Yamaguchi-Shinozaki K. Transcriptional regulatory network of plant heat stress response. Trends Plant Sci. 2017 22(1):53-65.

19. Gong Z, Xiong L, Shi H, Yang S, Herrera-Estrella LR, Xu G, Chao D-Y, Li J, Wang P-Y, Qin F, et al. Plant abiotic stress response and nutrient use efficiency. Sci China-Life Sci. 2020;63:635-74.

20. Wang W, Vinocur B, Shoseyov O, Altman A. Role of plant heat-shock proteins and molecular chaperones in the abiotic stress response. Trends Plant Sci. 2004;9(5):244-52.

21. Sarkar NK, Kim YK, Grover A. Rice sHsp genes: genomic organization and expression profiling under stress and development. BMC Genomics. 2009; 10(1):393.

22. Wen F, Wu X, Li T, Jia M, Liu X, Li P, Zhou X, Ji X, Yue X. Genome-wide survey of heat shock factors and heat shock protein 70s and their regulatory network under abiotic stresses in Brachypodium distachyon. PLoS One. 2017;12(7):e0180352.

23. Lin YX. Genome-wide identification, classification and analysis of heat shock transcription factor family in maize. BMC Genomics. 2011;12(1):76.

24. Kim KH. Overexpression of a chloroplast-localized small heat shock protein OsHSP26 confers enhanced tolerance against oxidative and heat stresses in tall fescue. Biotechnol Lett. 2012;34(2):371-7.

25. Mittal D, Chakrabarti S, Sarkar A, Singh A, Grover A. Heat shock factor gene family in rice: genomic organization and transcript expression profiling in response to high temperature, low temperature and oxidative stresses. Plant Physiol Biochem Ppb. 2009:47(9):785-95.

26. Liu HC, Liao HT, Charng YY. The role of class A1 heat shock factors (HSFA1s) in response to heat and other stresses in Arabidopsis. Plant Cell Environ. 2011;34(5):738-51. 
27. Yoshida T, Ohama N, Nakajima J, Kidokoro S, Mizoi J, Nakashima K, Maruyama K, Kim JM, Seki M, Todaka D, et al. Arabidopsis HsfA1 transcription factors function as the main positive regulators in heat shock-responsive gene expression. Mol Genet Genomics. 2011;286(5-6): 321-32.

28. Sato H, Mizoi J, Tanaka H, Maruyama K, Qin F, Osakabe Y, Morimoto K, Ohori T, Kusakabe K, Nagata M, et al. Arabidopsis DPB3-1, a DREB2A interactor, specifically enhances heat stress-induced gene expression by forming a heat stress-specific transcriptional complex with NF-Y subunits. Plant Cell. 2014;26(12):4954-73

29. Yoshida T, Sakuma Y, Todaka D, Maruyama K, Qin F, Mizoi J, Kidokoro S, Fujita Y, Shinozaki K, Yamaguchi-Shinozaki K. Functional analysis of an Arabidopsis heat-shock transcription factor HsfA3 in the transcriptional cascade downstream of the DREB2A stress-regulatory system. Biochem Biophys Res Commun. 2008;368(3):515-21.

30. Schramm F, Larkindale J, Kiehlmann E, Ganguli A, Englich G, Vierling E, von Koskull-Doring P. A cascade of transcription factor DREB2A and heat stress transcription factor $\mathrm{HsfA} 3$ regulates the heat stress response of Arabidopsis. Plant J. 2008;53(2):264-74.

31. Allakhverdiev SI, Kreslavski VD, Klimov W, Los DA, Carpentier R, Mohanty P. Heat stress: an overview of molecular responses in photosynthesis. Photosynth Res. 2008;98(1-3):541-50.

32. Kishor PK, Sangam S, Amrutha RN, Laxmi PS, Naidu KR, Rao K, Rao S, Reddy K, Theriappan P, Sreenivasulu N. Regulation of proline biosynthesis, degradation, uptake and transport in higher plants : Its implications in plant growth and abiotic stress tolerance. Curr Sci. 2005; 88(3):424-38.

33. Matysik J, Bhalu BA, Mohanty P, Bohrweg N. Molecular mechanism of quenching of reactive oxygen species by proline under stress in plants. Curr Sci. 2002;82(5):525-32.

34. Jiang $Y$, Huang B. Osmotic adjustment and root growth associated with drought preconditioning-enhanced heat tolerance in Kentucky bluegrass. Crop Sci. 2001;41(4):1168-73.

35. Ashraf M, Saeed MM, Qureshi MJ. Tolerance to high temperature in cotton (Gossypium hirsutum L.) at initial growth stages. Environ Exp Bot. 1994;34(3): 275-83.

36. Carretero-Paulet L, Galstyan A, Roig-Villanova I, Martinez-Garcia JF, BilbaoCastro JR, Robertson DL. Genome-wide classification and evolutionary analysis of the bHLH family of transcription factors in Arabidopsis, poplar, rice, moss, and algae. Plant Physiol. 2010;153(3):1398-412.

37. Duan S, Liu B, Zhang Y, Li G, Guo X. Genome-wide identification and abiotic stress-responsive pattern of heat shock transcription factor family in Triticum aestivum L. BMC Genomics. 2019;20(1):257.

38. Jakoby M, Weisshaar B, Dröge-Laser W, Vicente-Carbajosa J, Tiedemann J, Kroj T, FJTiPS P. bZIP transcription factors in Arabidopsis. Trends Plant Sci. 2002;7(3):106-11.

39. Zhao H, Wu D, Kong F, Lin K, Zhang H, Li G. The Arabidopsis thaliana nuclear factor $Y$ transcription factors. Front Plant Sci. 2016;7:2045.

40. Yan Q, Wu F, Ma T, Zong X, Ma Q, Li J, Zhao Y, Wang Y, Zhang J, Comprehensive analysis of bZIP transcription factors uncovers their roles during dimorphic floret differentiation and stress response in Cleistogenes songorica. BMC Genomics. 2019;20(1):760.

41. Jain M, Ghanashyam C, Bhattacharjee A: Comprehensive expression analysis suggests overlapping and specific roles of rice glutathione S-transferase genes during development and stress responses. BMC Genomics 2010, 11(1):1-17.

42. Chen JH, Jiang H-W, Hsieh E-J, Chen H-Y, Chien C-T, Hsieh H-L, Lin T-P. Drought and salt stress tolerance of an Arabidopsis Glutathione S-Transferase U17 knockout mutant are attributed to the combined effect of glutathione and abscisic acid. Plant Physiol. 2012;158(1):340-51.

43. Zwiewka M, Nodzyński T, Robert S, Vanneste S, Friml J. Osmotic stress modulates the balance between exocytosis and clathrin-mediated endocytosis in Arabidopsis thaliana. Mol Plant. 2015;8(8):1175-87.

44. Golani Y, Kaye Y, Gilhar O, Ercetin M, Gillaspy G, Levine A. Inositol polyphosphate phosphatidylinositol 5-Phosphatase9 (At5PTase9) controls plant salt tolerance by regulating endocytosis. Mol Plant. 2013;6(6): 1781-94.

45. Shen $Y_{1,}, X_{u} L_{1}$., Foster DA: Role for phospholipase $D$ in receptor-mediated endocytosis. Mol Cell Biol 2001, 21(2):595-602.

46. Bargmann BO, Munnik T. The role of phospholipase D in plant stress responses. Curr Opin Plant Biol. 2006;9(5):515-22.
47. Fischer I, Dievart A, Droc G. Dufayard J-Fo, Chantret N: evolutionary dynamics of the Leucine-rich repeats receptor-like kinase (LRR-RLK) subfamily in angiosperms. Plant Physiol. 2016;170(3):01470.02015.

48. Zhu JK. Abiotic stress signaling and responses in plants. Cell. 2016;167(2): 313-24.

49. Weston DJ, Karve AA, Gunter LE, Jawdy SS, Yang X, Allen SM, Wullschleger SD. Comparative physiology and transcriptional networks underlying the heat shock response in Populus trichocarpa, Arabidopsis thaliana and Glycine max. Plant Cell Environ. 2011;34(9):1488-506.

50. Scharf KD, Berberich T, Ebersberger I, Nover L. Corrigendum to "the plant heat stress transcription factor (Hsf) family: structure, function and evolution" [BBAGRM 1819 (2) 104-119]. Biochim Biophys Acta Gene Regul Mech. 2018;1861(1):60.

51. Hu W, Hu G, Han B. Genome-wide survey and expression profiling of heat shock proteins and heat shock factors revealed overlapped and stress specific response under abiotic stresses in rice. Plant Sci Int J Exp Plant Biol. 2009;176(4):583-90.

52. Shaar-Moshe L, Hubner S, Peleg Z. Identification of conserved droughtadaptive genes using a cross-species meta-analysis approach. BMC Plant Biol. 2015;15:111.

53. JALINK, Schoor VD: LED Induced chlorophyll fluorescence transient imager for measurements of health and stress status of whole plants. In: 2011; 2011.

54. Kim D, Langmead B, Salzberg SL. HISAT: a fast spliced aligner with low memory requirements. Nat Methods. 2015;12(4):357-60.

55. Pertea M, Pertea GM, Antonescu CM, Chang TC, Mendell JT, Salzberg SL. StringTie enables improved reconstruction of a transcriptome from RNA-seq reads. Nat Biotechnol. 2015;33(3):290-5.

56. Krzywinski M, Schein Jl. Circos: an information aesthetic for comparative genomics. Genome Res. 2009;19(9):1639-45.

57. Shaar-Moshe L, Blumwald E, Peleg Z. Unique physiological and transcriptional shifts under combinations of salinity, drought, and heat. Plant Physiol. 2017;174(1):421-34.

58. Li L, Stoeckert CJ Jr, Roos DS. OrthoMCL: identification of ortholog groups for eukaryotic genomes. Genome Res. 2003;13(9):2178-89.

59. Langfelder $P$, Horvath S. WGCNA: an R package for weighted correlation network analysis. Bmc Bioinformatics. 2008:9:559.

60. Nguyen LT, Schmidt HA, von Haeseler A, Minh BQ. IQ-TREE: a fast and effective stochastic algorithm for estimating maximum-likelihood phylogenies. Mol Biol Evol. 2015;32(1):268-74.

61. Wang Y, Tang H, Debarry JD, Tan X, Li J, Wang X, Lee TH, Jin H, Marler B, Guo H, et al. MCScanX: a toolkit for detection and evolutionary analysis of gene synteny and collinearity. Nucleic Acids Res. 2012;40(7):e49.

62. Livak KJ, Schmittgen TD. Analysis of relative gene expression data using real-time quantitative PCR and the 2(-Delta DeltaC(T)) Method. Methods (San Diego, Calif). 2001;25(4):402-8.

\section{Publisher's Note}

Springer Nature remains neutral with regard to jurisdictional claims in published maps and institutional affiliations.

Ready to submit your research? Choose BMC and benefit from:

- fast, convenient online submission

- thorough peer review by experienced researchers in your field

- rapid publication on acceptance

- support for research data, including large and complex data types

- gold Open Access which fosters wider collaboration and increased citations

- maximum visibility for your research: over $100 \mathrm{M}$ website views per year

At $\mathrm{BMC}$, research is always in progress.

Learn more biomedcentral.com/submission 